TRANSACTIONS OF THE

AMERICAN MATHEMATICAL SOCIETY

Volume 363, Number 1, January 2011, Pages 545-577

S 0002-9947(2010)05268-7

Article electronically published on August 31, 2010

\title{
EXTENDING POSITIVE DEFINITENESS
}

\author{
DARIUSZ CICHOŃ, JAN STOCHEL, AND FRANCISZEK HUGON SZAFRANIEC
}

To the memory of M.G. Krein (1907-1989)

\begin{abstract}
The main result of this paper gives criteria for extendibility of mappings defined on symmetric subsets of $*$-semigroups to positive definite ones. By specifying the mappings in question we obtain new solutions of relevant issues in harmonic analysis concerning truncations of some important multivariate moment problems, like complex, two-sided complex and multidimensional trigonometric moment problems. In addition, unbounded subnormality and existence of unitary power dilation of several contractions is treated as an application of our general scheme.
\end{abstract}

\section{INTRODUCTION}

In 61] a fairly general concept of $*$-semigroups, which includes groups, $*$-algebras and quite a number of instances in between, as well as positive definite functions on them, has been originated by Sz.-Nagy on occasion of his general dilation theorem. On the other hand, the parallel notion of complete positivity, which is involved in the so-called Stinespring dilation theorem, turns out to be equivalent to the former; cf. 59. This is so as long as bounded operators are on the dilation level. However, Sz.-Nagy's approach offers a much more useful environment to work in, which is apparent when dealing with moment problems.

If one goes beyond bounded operator dilations, the two notions, positive definiteness and complete positivity, still make sense but are no longer equivalent. This happens especially when one deals with moment problems on unbounded sets. In this case positivity understood as in the sense of Marcel Riesz and Haviland [43, 28, 29] plays a role, too. Therefore, there is a need for common treatment of these by means of forms over *-semigroups, as in [58. The aforesaid cases are represented in our paper by unbounded subnormal operators and the complex

Received by the editors December 22, 2008 and, in revised form, December 9, 2009.

2010 Mathematics Subject Classification. Primary 43A35, 44A60; Secondary 47A20, 47B20.

Key words and phrases. Positive definite mapping, completely positive mapping, completely f-positive mapping, complex moment problem, multidimensional trigonometric moment problem, truncated moment problems, sums of squares (SOS), the 17th Hilbert problem, subnormal operator, unitary power dilation for several contractions.

This work was partially supported by KBN grant 2 P03A 037024 and by MNiSzW grant N201 026 32/1350. The third author would like to acknowledge assistance of the EU Sixth Framework Programme for the Transfer of Knowledge "Operator theory methods for differential equations" (TODEQ) \# MTKD-CT-2005-030042. A very early version of this paper was designated for Kreĭn's anniversary volume. However, due to its growing capacity we have been exceeding all consecutive deadlines; let us thank Professor Vadim Adamyan for his patience in negotiating them. 
moment problem. In addition to this we also consider the "bounded" case of unitary dilations of several operators and, what is related to, of the multidimensional trigonometric moment problem.

A topic attracting the attention of quite a number of mathematicians is the extendibility of functions to either positive definite or completely positive ones. The most classical result in this matter concerns groups. It states that every continuous positive definite function on a closed subgroup of a locally compact abelian (or compact, not necessarily abelian) group extends to a continuous positive definite function on the whole group (cf. [30, Section 34.48(a)\&(c)] and [15, Theorem 3.16]). However, leaving the topological requirements aside, each positive definite function on a subgroup of a group $G$ extends by zero to a positive definite function on the whole $G$ (cf. [30, Section 32.43(a)]). This procedure is no longer applicable to *semigroups other than groups. What is more, not every positive definite function on a $*$-subsemigroup extends to a positive definite function on the whole $*$-semigroup. This is best exemplified by the interplay between the $*$-semigroups $\mathfrak{N} \subset \mathfrak{N}_{+}$as shown in [54] in connection with the complex moment problem (see Example 3 and Section 5 for the definitions).

The situation becomes more complicated when one wants to extend positive definiteness from subsets of a more relaxed structure, even in the case of groups. The classical result of Krein 35] on automatic extendibility of continuous positive definite functions from a symmetric interval to the whole real line suggests that symmetricity of the subset may be essential. This is somehow confirmed by [55], which contains a full characterization of several contractions having commuting unitary dilations. For more discussion of the role played by symmetry, we refer to Section 5.1. The results contained in [3, 4] and [14] also corroborate the importance of symmetry; the latter concerns extensions to indefinite forms with a finite number of negative squares.

One of the main ideas of the present paper is to employ generalized polynomial functions to the extendibility criteria invented in [54] and [55]. What we get is strictly related to complete positivity of associated linear mappings. The original contribution consists of introducing complete f-positivity (cf. Theorems 14 and 15). This results, in particular, in the complex variant of the Riesz-Haviland theorem (cf. Theorem 19); the complete f-positivity is now written in terms of positivity of the associated linear functional on the set of all finite sums of squares of moduli of very special rational functions in variables $z$ and $\bar{z}$.

Carefully selected applications are chosen as follows. Considering determining subsets of $\mathfrak{X}_{\mathfrak{N}_{+}}$allows us to apply Theorems 14 and 15 to the complex moment problem (Theorem 19) as well as to subnormal operators (Theorem 21). Theorem 19 can be thought of as a truncated moment problem, however, not in the usual sense of finite sections. Analogous results are formulated for the truncated multidimensional trigonometric moment problem and the truncated two-sided complex moment problem (cf. Theorems 26 and 32). Moreover, Theorem 24 contains a new characterization of finite systems of Hilbert space operators admitting unitary power dilations.

Section 9 deals with inter alia, approximation of nonnegative polynomials in indeterminates $z$ and $\bar{z}$ by sums of finitely many squares of moduli of rational functions that are linear combinations of expressions $z^{m} \bar{z}^{n}$ with $m+n \geqslant 0$ (cf. Proposition 28). This can be compared to Artin's solution of the 17th Hilbert problem stating 
that every nonnegative polynomial in $z$ and $\bar{z}$ is a sum of finitely many squares of moduli of (a priori arbitrary) rational functions. The above approximation is no longer possible when considered on proper closed subsets of $\mathbb{C}$; cf. Proposition 20 (see also Proposition 27 for the case of multivariable trigonometric polynomials). Similar approximation holds for multivariable trigonometric polynomials (cf. Proposition [30). A more detailed discussion relating the theme to selected recent articles [20, 27] is contained in Section 9.

\section{GenerAL CRITERIA}

Besides keeping $\mathbb{Z}, \mathbb{R}, \mathbb{C}$ for standard sets of integer, real and complex numbers, respectively, by $\mathbb{Z}_{+}$we understand the set $\{0,1,2, \ldots\}$. Moreover, we adopt the notation $\mathbb{T}=\{z \in \mathbb{C}:|z|=1\}$ and $\mathbb{C}_{*}=\mathbb{C} \backslash\{0\}$. A system $\left\{z_{\omega}\right\}_{\omega \in \Omega}$ of complex numbers is said to be finite if the set $\left\{\omega \in \Omega: z_{\omega} \neq 0\right\}$ is finite.

1. Polynomials on a dual $*$-semigroup. Given a nonempty set $\Omega$, we denote by $\mathbb{C}^{\Omega}$ the complex $*$-algebra of all complex functions on $\Omega$ with the algebra operations defined pointwisely and the involution

$$
f^{*}(\omega)=\overline{f(\omega)}, \quad f \in \mathbb{C}^{\Omega}, \omega \in \Omega
$$

The following fact reveals the idea standing behind the known characterization of linear independence of Laplace transforms of elements of a commutative semigroup (see [31] and [5, Proposition 6.1.8] for a pattern of the proof).

Lemma 1. Let $\Omega$ be a nonempty set and let $Y \subset \mathbb{C}^{\Omega}$ be a semigroup (with respect to the multiplication of $\mathbb{C}^{\Omega}$ ) containing the constant function 1 . Then the following conditions are equivalent:

(i) $Y$ separates points of $\Omega$ (equivalently: if $\left.\widehat{\omega_{1}}\right|_{Y}=\left.\widehat{\omega_{2}}\right|_{Y}$, then $\omega_{1}=\omega_{2}$ ),

(ii) the system $\left\{\left.\widehat{\omega}\right|_{Y}\right\}_{\omega \in \Omega}$ is linearly independent in $\mathbb{C}^{Y}$,

where for $\omega \in \Omega$ the function $\widehat{\omega}: \mathbb{C}^{\Omega} \rightarrow \mathbb{C}$ is defined by $\widehat{\omega}(f)=f(\omega), f \in \mathbb{C}^{\Omega}$.

If the $Y$ in Lemma 1 is not semigroup, then the implication (i) $\Longrightarrow$ (ii) may no longer be true (cf. Example 33).

As far as abstract semigroups are concerned, we adhere to the multiplicative notation. A mapping $\mathfrak{S} \ni s \longmapsto s^{*} \in \mathfrak{S}$ defined on a semigroup $\mathfrak{S}$ is called an involution if $(s t)^{*}=t^{*} s^{*}$ and $\left(s^{*}\right)^{*}=s$ for all $s, t \in \mathfrak{S}$. A semigroup $\mathfrak{S}$ equipped with an involution is said to be a $*$-semigroup. It is clear that if $\mathfrak{S}$ has a unit $\varepsilon$, then $\varepsilon^{*}=\varepsilon$. The set $\left\{s \in \mathfrak{S}: s=s^{*}\right\}$ of all Hermitian elements of a $*$-semigroup $\mathfrak{S}$ is denoted by $\mathfrak{S}_{\mathfrak{h}}$. For a nonempty subset $T$ of a $*$-semigroup $\mathfrak{S}$, we write $T^{*}=\left\{s^{*}: s \in T\right\} ; T$ is said to be symmetric if $T=T^{*}$. Put $[T]=\bigcup_{n=1}^{\infty} T^{[n]}$, where $T^{[n]}$ stands for the set of all products of length $n$ with factors in $T$. The set $[T]$ is the smallest subsemigroup of $\mathfrak{S}$ containing $T$. Under the assumption of commutativity of $\mathfrak{S}$, the set $\llbracket T \rrbracket \stackrel{\text { def }}{=}\left\{u^{*} v: u, v \in[T]\right\}$ is a $*$-subsemigroup of $\mathfrak{S}$ which does not have to contain any of the sets $T$ and $T^{*}$. Neither $[T]$ nor $\llbracket T \rrbracket$ has to contain the unit of $\mathfrak{S}$ even if it exists. 
Let $\mathfrak{S}$ be a commutative $*$-semigroup with a unit $\varepsilon$. A function $\chi: \mathfrak{S} \rightarrow \mathbb{C}$ is called a character of $\mathfrak{S}$ if

$$
\begin{aligned}
& \chi(s t)=\chi(s) \chi(t), \quad s, t \in \mathfrak{S}, \\
& \chi\left(s^{*}\right)=\overline{\chi(s)}, \quad s \in \mathfrak{S}, \\
& \chi(\varepsilon)=1 .
\end{aligned}
$$

The set $\mathfrak{X}_{\mathfrak{S}}$ of all characters of $\mathfrak{S}$ is a *-semigroup with respect to the multiplication and the involution of $\mathbb{C}^{\mathfrak{S}} ; \mathfrak{X}_{\mathfrak{S}}$ is called a dual $*$-semigroup of $\mathfrak{S}$. For every $s \in \mathfrak{S}$, we define the function $\hat{s}: \mathfrak{X}_{\mathfrak{S}} \rightarrow \mathbb{C}$, modelled on the Fourier, Gelfand or Laplace transform, via

$$
\hat{s}(\chi)=\chi(s), \quad \chi \in \mathfrak{X}_{\mathfrak{S}} .
$$

The set $\{\hat{s}: s \in \mathfrak{S}\}$ will be denoted by $\widehat{\mathfrak{S}}$. It follows from (11), (2) and (3) that $\widehat{s t}=\hat{s} \cdot \hat{t}$ and $\widehat{s^{*}}=\overline{\hat{s}}$ for all $s, t \in \mathfrak{S}$, and $\hat{\varepsilon} \equiv 1$. This means that $\widehat{\mathfrak{S}}$ is a $*$-semigroup with respect to the multiplication and the involution of $\mathbb{C}^{\mathfrak{X}_{\mathfrak{G}}}$.

The set $\mathcal{F}_{\mathfrak{S}}$ of all complex functions on $\mathfrak{S}$ vanishing off finite sets is a complex *algebra with pointwise defined linear algebra operations, the algebra multiplication of convolution type

$$
(f \star g)(u)=\sum_{\substack{s, t \in \mathfrak{S} \\ u=s t}} f(s) g(t), \quad f, g \in \mathcal{F}_{\mathfrak{S}}, u \in \mathfrak{S},
$$

and the involution

$$
f^{*}(s)=\overline{f\left(s^{*}\right)}, \quad f \in \mathcal{F}_{\mathfrak{S}}, s \in \mathfrak{S} .
$$

For a nonempty subset $T$ of $\mathfrak{S}$, we define the linear subspace $\mathcal{F}_{\mathfrak{S}, T}$ of $\mathcal{F}_{\mathfrak{S}}$ via

$$
\mathcal{F}_{\mathfrak{S}, T}=\left\{f \in \mathcal{F}_{\mathfrak{S}}: f \text { vanishes off the set } T\right\} .
$$

With the algebra operations defined above, $\mathcal{F}_{\mathfrak{S}, T}$ is a subalgebra of $\mathcal{F}_{\mathfrak{S}}$ if and only if $T$ is a subsemigroup of $\mathfrak{S}$; what is more, $\mathcal{F}_{\mathfrak{S}, T}$ is a symmetric subset of $\mathcal{F}_{\mathfrak{S}}$ if and only if $T$ is a symmetric subset of $\mathfrak{S}$. The reader should be aware of the fact that if $\mathfrak{S}$ is finite, then although the sets $\mathcal{F}_{\mathfrak{S}}$ and $\mathbb{C}^{\mathfrak{S}}$ coincide, their $*$-algebra structures differ unless $\mathfrak{S}$ is a singleton.

Given a nonempty subset $Y$ of $\mathfrak{X}_{\mathfrak{S}}$, we write $\mathscr{P}(Y)$ for the linear span of $\left\{\left.\hat{s}\right|_{Y}: s \in\right.$ $\mathfrak{S}\}$ in $\mathbb{C}^{Y}$. Clearly, $\mathscr{P}(Y)$ is a $*$-subalgebra of the $*$-algebra $\mathbb{C}^{Y}$. Notice that there exists a unique $*$-algebra epimorphism $\Delta_{Y}: \mathcal{F}_{\mathfrak{S}} \rightarrow \mathscr{P}(Y)$ such that

$$
\Delta_{Y}\left(\delta_{s}\right)=\left.\hat{s}\right|_{Y}, \quad s \in \mathfrak{S},
$$

where $\delta_{s} \in \mathcal{F}_{\mathfrak{S}}$ is the characteristic function of $\{s\}$. For a nonempty subset $T$ of $\mathfrak{S}$, we write $\mathscr{P}_{T}(Y)=\Delta_{Y}\left(\mathcal{F}_{\mathfrak{S}, T}\right)$; the linear space $\mathscr{P}_{T}(Y)$ coincides with the linear span of $\left\{\left.\hat{s}\right|_{Y}: s \in T\right\}$.

In this paper we are interested in the case in which the $*$-algebra homomorphism $\Delta_{Y}$ is injective. The following is a direct consequence of Lemma 1.

Proposition 2. If $Y$ is a subsemigroup of $\mathfrak{X}_{\mathfrak{S}}$ containing the constant function 1, then the following conditions are equivalent:

(i) $\Delta_{Y}$ is a *-algebra isomorphism,

(ii) the system $\left\{\left.\hat{s}\right|_{Y}\right\}_{s \in \mathfrak{S}}$ is linearly independent in $\mathbb{C}^{Y}$,

(iii) $Y$ separates the points of $\mathfrak{S}$ (equivalently: if $\left.\hat{s}\right|_{Y}=\left.\hat{t}\right|_{Y}$, then $s=t$ ). 
2. Determining sets. A nonempty subset $Y$ of $\mathfrak{X}_{\mathfrak{S}}$ is called determining (for $\mathscr{P}\left(\mathfrak{X}_{\mathfrak{S}}\right)$ ) if it satisfies any of the equivalent conditions (i) and (ii) of Proposition 2 If a subset $Y$ of $\mathfrak{X}_{\mathfrak{S}}$ is determining, then the mapping

$$
\pi_{T, Y}:\left.\mathscr{P}_{T}\left(\mathfrak{X}_{\mathfrak{S}}\right) \ni w \mapsto w\right|_{Y} \in \mathscr{P}_{T}(Y)
$$

is a well defined $*$-algebra isomorphism (but not conversely; see the next paragraph).

It may happen that the whole dual $*$-semigroup $\mathfrak{X}_{\mathfrak{S}}$ does not separate the points of $\mathfrak{S}$, and consequently $\mathfrak{X}_{\mathfrak{S}}$ is not determining (cf. [5, Remarks 4.6.9(1)]). If $Y$ is not a subsemigroup of $\mathfrak{X}_{\mathfrak{S}}$, then implication (iii) $\Longrightarrow$ (ii) of Proposition 2 may fail to hold (implication (ii) $\Longrightarrow$ (iii) is always true). To see this, we shall discuss a *-semigroup introduced in 1955 by Sz.-Nagy 61] for which the set $\mathscr{P}\left(\mathfrak{X}_{\mathfrak{S}}\right)$ can be interpreted as the ring of all polynomials in $z$ and $\bar{z}$.

Example 3. Equip the Cartesian product $\mathbb{Z}_{+} \times \mathbb{Z}_{+}$with coordinatewise defined addition as a semigroup operation, i.e. $(i, j)+(k, l)=(i+k, j+l)$, and involution $(m, n)^{*}=(n, m)$. The $*$-semigroup so obtained will be denoted by $\mathfrak{N}$. If $\mathbb{C}$ is thought of as a *-semigroup with multiplication as a semigroup operation and complex conjugation as an involution, then the mapping

$$
\mathfrak{X}_{\mathfrak{N}} \ni \chi \mapsto \chi(1,0) \in \mathbb{C},
$$

being a *-semigroup isomorphism, enables us to identify algebraically $\mathfrak{X}_{\mathfrak{N}}$ with $\mathbb{C}$. Under this identification, we have

$$
\widehat{(m, n)}(z)=z^{m} \bar{z}^{n}, \quad m, n \in \mathbb{Z}_{+}, z \in \mathbb{C} .
$$

Note that by (44) and Lemma 5 below the system $\{\widehat{(m, n)}\}_{(m, n) \in \mathfrak{N}}$ is linearly independent in $\mathbb{C}^{\mathbb{C}}$. Hence, the set $\mathscr{P}\left(\mathfrak{X}_{\mathfrak{N}}\right)$ can be thought of as the ring $\mathbb{C}[z, \bar{z}]$ of all complex polynomials in $z$ and $\bar{z}$.

It turns out that it is possible to give a satisfactory description of all subsets of $\mathbb{C}$ separating the points of $\mathfrak{N}$.

Proposition 4. A subset $Y$ of $\mathbb{C}$ separates the points of $\mathfrak{N}$ if and only if the following two conditions hold:

(i) $Y \not \subset \mathbb{T} \cup\{0\}$,

(ii) $Y_{1} \stackrel{\text { def }}{=}\left\{\frac{z}{|z|}: z \in Y \backslash\{0\}\right\} \not \subset\left\{w \in \mathbb{C}: w^{\varkappa}=1\right\}$ for every integer $\varkappa \geqslant 1$.

Proof. Suppose that $Y$ does not satisfy the conjunction of conditions (i) and (ii). Then either $Y \subset \mathbb{T} \cup\{0\}$, and hence $z^{2} \bar{z}=z$ for all $z \in Y$, or $Y_{1} \subset\left\{w \in \mathbb{C}: w^{\varkappa}=1\right\}$ for some $\varkappa \geqslant 1$, and hence $z^{2 \varkappa}=z^{\varkappa} \bar{z}^{\varkappa}$ for all $z \in Y$. In both cases, $Y$ cannot separate the points of $\mathfrak{N}$.

Assume now that $Y$ satisfies (i) and (ii). Take $m, n, k, l \in \mathbb{Z}_{+}$and suppose that $z^{m} \bar{z}^{n}=z^{k} \bar{z}^{l}$ for all $z \in Y$. Taking absolute value of both sides of the equality and employing (i) we get

$$
j \stackrel{\text { def }}{=} m+n=k+l
$$

Dividing both sides of $z^{m} \bar{z}^{n}=z^{k} \bar{z}^{l}$ by $|z|^{j}$ gives $w^{\varkappa}=1$ for all $w \in Y_{1}$ with $\varkappa=m-n-(k-l)$. By (ii) this implies that $\varkappa=0$, which when combined with (5) leads to $(m, n)=(k, l)$. The proof is complete. 
We now indicate a class of subsets $Y$ of $\mathfrak{X}_{\mathfrak{N}}$ which separate the points of $\mathfrak{N}$ but which are not determining for $\mathscr{P}\left(\mathfrak{X}_{\mathfrak{N}}\right)=\mathbb{C}[z, \bar{z}]$. Take a nonzero polynomial $p \in \mathbb{C}[z, \bar{z}]$ such that the set

$$
Y_{p} \stackrel{\text { def }}{=}\{z \in \mathbb{C}: p(z, \bar{z})=0\}
$$

is nonempty. Then evidently the functions $\left\{\left.\widehat{(m, n)}\right|_{Y_{p}}: m, n \in \mathbb{Z}_{+}\right\}$are linearly dependent in $\mathbb{C}^{Y_{p}}$, and hence $Y_{p}$ is not determining for $\mathbb{C}[z, \bar{z}]$. Let us focus on the case of circles and straight lines (which are always of the form ([6]). It follows from Proposition 4 that the ensuing sets do not separate the points of $\mathfrak{N}$ :

- the unit circle $\mathbb{T}$ centered at the origin,

- a straight line $L$ such that $0 \in L$, and the points of $L \cap \mathbb{T}$ are complex $\varkappa$-roots of 1 for some (necessarily even) integer $\varkappa \geqslant 2$.

All the other circles and straight lines do separate the points of $\mathfrak{N}$ (in many cases they embrace 1). Surprisingly, one point sets, which are still of the form (6), may separate the points of $\mathfrak{N}$. Indeed, by Proposition 4 if $z \in \mathbb{C} \backslash(\mathbb{T} \cup\{0\})$ and for any integer $\varkappa \geqslant 1, \frac{z}{|z|}$ is not a complex $\varkappa$-root of 1 , then $\{z\}$ does separate the points of $\mathfrak{N}$.

Contrary to the case of sets separating the points of $\mathfrak{N}$, one cannot expect any neat description of all determining set $\mathbb{1}^{1}$ for $\mathbb{C}[z, \bar{z}]$. Nevertheless, we may indicate some determining sets explicitly.

Lemma 5. Suppose that $Y \subset \mathbb{C}$ is either a union of infinitely many parallel straight lines or a union of infinitely many concentric circles. If $\left\{a_{m, n}\right\}_{m, n \in \mathbb{Z}}$ is a finite system of complex numbers such that $\sum_{m, n \in \mathbb{Z}} a_{m, n} z^{m} \bar{z}^{n}=0$ for all $z \in Y \backslash\{0\}$, then $a_{m, n}=0$ for all $m, n \in \mathbb{Z}$.

Proof. Since the set $\left\{(m, n) \in \mathbb{Z} \times \mathbb{Z}: a_{m, n} \neq 0\right\}$ is finite, there exists an integer $N \geqslant 1$ such that $a_{m, n}=0$ for all integers $m, n$ such that $m<-N$ or $n<-N$. This implies that $p(z)=\sum_{m, n \geqslant-N} a_{m, n} z^{m+n} \bar{z}^{n+N}$ is a complex polynomial in $z$ and $\bar{z}$ vanishing on $Y$. In the case of straight lines, we can always find $\theta \in(-\pi / 2, \pi / 2]$ such that the polynomial $p\left(\mathrm{e}^{\mathrm{i} \theta} z\right)$ vanishes on a union of infinitely many straight lines parallel to the real axis. Next, considering the complex polynomial $p\left(\mathrm{e}^{\mathrm{i} \theta}(x+\mathrm{i} y)\right)$ in two real variables $x$ and $y$, we deduce that $p(z)=0$ for all $z \in \mathbb{C}$. In the other case, applying a suitable translation of the argument, we can assume that the common center of the circles is in the origin. Employing a well known identity principle for complex polynomials in $z$ and $\bar{z}$ completes the proof in both cases.

3. Complete positivity. Let $\mathfrak{S}$ be a unital commutative $*$-semigroup, $T$ a nonempty subset of $\mathfrak{S}$ and $Y$ a nonempty subset of $\mathfrak{X}_{\mathfrak{S}}$. Denote by $\mathscr{P}_{T}\left(Y, \ell^{2}\right)$ the set of all functions from $Y$ to $\ell^{2}$ of the form $\chi \mapsto \sum_{s \in T} \chi(s) x_{s}$, where $\left\{x_{s}\right\}_{s \in T} \subset \ell^{2}$ and $x_{s}=0$ for all but a finite number of $s$ 's. Note that $\mathscr{P}_{T}\left(Y, \ell^{2}\right)$ can be thought of as the algebraic tensor product $\mathscr{P}_{T}(Y) \otimes \ell^{2}$. We abbreviate $\mathscr{P}_{\mathfrak{S}}\left(Y, \ell^{2}\right)$ to $\mathscr{P}\left(Y, \ell^{2}\right)$. The standard notation $\left[a_{k, l}\right]_{k, l=1}^{m} \geqslant 0$ is used for nonnegativity of the scalar matrix $\left[a_{k, l}\right]_{k, l=1}^{m}$.

For the linear space

$$
\mathrm{M}^{m}\left(\mathscr{P}_{T}(Y)\right) \stackrel{\text { def }}{=}\left\{\left[w_{k, l}\right]_{k, l=1}^{m}: w_{k, l} \in \mathscr{P}_{T}(Y) \text { for all } k, l=1, \ldots, m\right\}, \quad m \geqslant 1,
$$

${ }^{1}$ It is clear that a subset $Z$ of $\mathbb{C}$ is determining for $\mathbb{C}[z, \bar{z}]$ if and only if $Z$ is dense in $\mathbb{R}^{2}$ with respect to the Zariski topology of $\mathbb{R}^{2}$ (see [13] for the necessary background). 
its subsets

$$
\mathrm{M}_{+}^{m}\left(\mathscr{P}_{T}(Y)\right) \stackrel{\text { def }}{=}\left\{\left[w_{k, l}\right]_{k, l=1}^{m} \in \mathrm{M}^{m}\left(\mathscr{P}_{T}(Y)\right):\left[w_{k, l}(\chi)\right]_{k, l=1}^{m} \geqslant 0 \text { for all } \chi \in Y\right\}
$$

and

$$
\mathrm{M}_{\mathrm{f}}^{m}\left(\mathscr{P}_{T}(Y)\right) \stackrel{\text { def }}{=} \mathrm{M}^{m}\left(\mathscr{P}_{T}(Y)\right) \cap\left\{\left[\left\langle p_{k}(\cdot), p_{l}(\cdot)\right\rangle_{\ell^{2}}\right]_{k, l=1}^{m}: p_{1}, \ldots, p_{m} \in \mathscr{P}\left(Y, \ell^{2}\right)\right\},
$$

where $\left\langle p_{k}(\cdot), p_{l}(\cdot)\right\rangle_{\ell^{2}}$ is the function $\chi \mapsto\left\langle p_{k}(\chi), p_{l}(\chi)\right\rangle_{\ell^{2}}$, turn out to be convex cones. Note that if $p_{1}, \ldots, p_{m} \in \mathscr{P}\left(Y, \ell^{2}\right)$, then $\left[\left\langle p_{k}(\cdot), p_{l}(\cdot)\right\rangle_{\ell^{2}}\right]_{k, l=1}^{m} \in \mathrm{M}_{+}^{m}(\mathscr{P}(Y))$. This implies that $\mathrm{M}_{\mathrm{f}}^{m}\left(\mathscr{P}_{T}(Y)\right) \subset \mathrm{M}_{+}^{m}\left(\mathscr{P}_{T}(Y)\right)$. One can also check that the square of each matrix from $\mathrm{M}_{\mathrm{f}}^{m}\left(\mathscr{P}_{T}(Y)\right)$ (respectively $\mathrm{M}_{+}^{m}\left(\mathscr{P}_{T}(Y)\right)$ ) belongs to $\mathrm{M}_{\mathrm{f}}^{m}\left(\mathscr{P}_{T^{[2]}}(Y)\right)$ (respectively $\mathrm{M}_{+}^{m}\left(\mathscr{P}_{T^{[2]}}(Y)\right)$; for $T^{[2]}$ go back to p. 547). What is more, if $Y$ is a determining subset of $\mathfrak{X}_{\mathfrak{S}}$, then the mappings

$$
\begin{gathered}
\left.\mathscr{P}\left(\mathfrak{X}_{\mathfrak{S}}, \ell^{2}\right) \ni p \mapsto p\right|_{Y} \in \mathscr{P}\left(Y, \ell^{2}\right), \\
\pi_{T, Y}^{(m)}: \mathrm{M}^{m}\left(\mathscr{P}_{T}\left(\mathfrak{X}_{\mathfrak{S}}\right)\right) \ni\left[w_{k, l}\right]_{k, l=1}^{m} \longmapsto\left[\left.w_{k, l}\right|_{Y}\right]_{k, l=1}^{m} \in \mathrm{M}^{m}\left(\mathscr{P}_{T}(Y)\right),
\end{gathered}
$$

are linear isomorphisms, and consequently

$$
\pi_{T, Y}^{(m)}\left(\mathrm{M}_{\mathrm{f}}^{m}\left(\mathscr{P}_{T}\left(\mathfrak{X}_{\mathfrak{S}}\right)\right)\right)=\mathrm{M}_{\mathrm{f}}^{m}\left(\mathscr{P}_{T}(Y)\right) .
$$

Lemma 6. If $p_{1}, \ldots, p_{m} \in \mathscr{P}\left(Y, \ell^{2}\right)$ and $A=\left[\left\langle p_{k}(\cdot), p_{l}(\cdot)\right\rangle_{\ell^{2}}\right]_{k, l=1}^{m}$, then there exists a matrix $P=\left[p_{k, j}\right]_{k=1}^{m=1}$ with entries $p_{k, j} \in \mathscr{P}(Y)$ such that $A=P P^{*}$, where $P^{*}=\left[\bar{p}_{j, k}\right]_{k=1}^{n} \prod_{j=1}^{m}$. Conversely, if $P$ is a matrix of size $m \times n$ with entries in $\mathscr{P}(Y)$, then $P P^{*}=\left[\left\langle p_{k}(\cdot), p_{l}(\cdot)\right\rangle_{\ell^{2}}\right]_{k, l=1}^{m}$ with some $p_{1}, \ldots, p_{m} \in \mathscr{P}\left(Y, \ell^{2}\right)$. In particular, if $p \in \mathscr{P}\left(Y, \ell^{2}\right)$, then $\langle p(\cdot), p(\cdot)\rangle_{\ell^{2}}=\sum_{j=1}^{n}\left|q_{j}\right|^{2}$ with some $q_{j} \in \mathscr{P}(Y)$.

Proof. Take $p_{1}, \ldots, p_{m} \in \mathscr{P}\left(Y, \ell^{2}\right)$. Then there exists a finite orthonormal basis $\left\{e_{j}\right\}_{j=1}^{n}$ of the linear span of $\bigcup_{k=1}^{m} p_{k}(Y)$. As a consequence, $p_{k}=\sum_{j=1}^{n} p_{k, j} e_{j}$ with some $p_{k, j} \in \mathscr{P}(Y)$, and hence $P=\left[p_{k, j}\right]_{k=1}^{m}{ }_{j=1}^{n}$ is the required matrix. Reversing the above reasoning concludes the proof.

We now examine the behavior of the classes $\mathrm{M}_{\mathrm{f}}^{m}\left(\mathscr{P}_{T}(Y)\right)$ and $\mathrm{M}_{+}^{m}\left(\mathscr{P}_{T}(Y)\right)$ under the operation of transposing their members.

Lemma 7. (i) If a matrix $\left[w_{k, l}\right]_{k, l=1}^{m}$ is a member of $\mathrm{M}_{+}^{m}\left(\mathscr{P}_{T}(Y)\right)$, then the transposed matrix $\left[w_{l, k}\right]_{k, l=1}^{m}$ belongs to $\mathrm{M}_{+}^{m}\left(\mathscr{P}_{T^{*}}(Y)\right)$.

(ii) If $p_{1}, \ldots, p_{m} \in \mathscr{P}_{T}\left(Y, \ell^{2}\right)$, then there exist $q_{1}, \ldots, q_{m} \in \mathscr{P}_{T^{*}}\left(Y, \ell^{2}\right)$ such that

$$
\left\langle p_{l}(\cdot), p_{k}(\cdot)\right\rangle_{\ell^{2}}=\left\langle q_{k}(\cdot), q_{l}(\cdot)\right\rangle_{\ell^{2}}, \quad k, l=1, \ldots, m .
$$

Proof. (i) By nonnegativity of $\left[w_{k, l}\right]_{k, l=1}^{m}$, we have $w_{l, k}(\chi)=\overline{w_{k, l}(\chi)}$ for all $\chi \in Y$ and $k, l=1, \ldots, m$. Hence, the application of (2) justifies (i).

(ii) Arguing as in the proof of Lemma 6, we can write $p_{k}=\sum_{j=1}^{n} p_{k, j} e_{j}$ with some $p_{k, j} \in \mathscr{P}_{T}(Y)$. Then

$$
\left\langle p_{l}(\chi), p_{k}(\chi)\right\rangle_{\ell^{2}}=\sum_{j=1}^{n} p_{l, j}(\chi) \overline{p_{k, j}(\chi)}=\left\langle q_{k}(\chi), q_{l}(\chi)\right\rangle_{\ell^{2}}, \quad \chi \in Y,
$$

where $q_{k}(\chi)=\sum_{j=1}^{n} \overline{p_{k, j}(\chi)} e_{j}$ for $\chi \in Y$. By (2), the functions $Y \ni \chi \mapsto \overline{p_{k, j}(\chi)} \in$ $\mathbb{C}$ belong to $\mathscr{P}_{T^{*}}(Y)$. This completes the proof. 
Let $F$ be a topological linear space and $\tau$ be its topology. The closure of a subset $W$ of $F$ with respect to $\tau$ is denoted by $\bar{W}^{\tau}$. Given an integer $m \geqslant 1$, we write $\mathrm{M}^{m}(F)$ for the linear space (with entrywise defined linear operations) of all $m$ by $m$ matrices with entries in $F$. Identifying $\mathrm{M}^{m}(F)$ with the Cartesian product of $m^{2}$ copies of $F$, we may regard $\mathrm{M}^{m}(F)$ as a topological linear space with the product topology $\tau^{(m)}$.

Call a nonempty subset $Z$ of $\mathfrak{X}_{\mathfrak{S}} T$-bounded ${ }^{2}$ if $\sup _{\chi \in Z}|\chi(s)|<\infty$ for every $s \in$ $T$. It is obvious that a nonempty subset $Z$ of $\mathfrak{X}_{\mathfrak{S}}$ is $T$-bounded if and only if it is $\langle T\rangle$ bounded, where $\langle T\rangle$ stands for the unital *-semigroup generated by $T$. Note also that nonempty $Z \subset \mathfrak{X}_{\mathfrak{S}}$ is $T$-bounded if and only if for every (equivalently: for some) integer $m \geqslant 1$ and for every $w \in \mathrm{M}^{m}\left(\mathscr{P}_{T}(Z)\right), \sup _{\chi \in Z}\|w(\chi)\|<\infty$; here $\|w(\chi)\|$ stands for the operator norm of the matrix $w(\chi)$. Denote by $\tau_{T, Y}$ the locally convex topology on $\mathscr{P}_{T}(Y)$ given by the system of seminorms $w \mapsto \sup _{\chi \in Z}|w(\chi)|$ indexed by $T$-bounded subsets $Z$ of $Y$. Observe that the topology $\tau_{T, Y}^{(m)}$ on $\mathrm{M}^{m}\left(\mathscr{P}_{T}(Y)\right)$ is identical with the locally convex topology given by the system of seminorms $w \mapsto \sup _{\chi \in Z}\|w(\chi)\|$, with $Z$ ranging over all $T$-bounded subsets of $Y$. It is clear that the topology $\tau_{T, Y}^{(m)}$ is stronger than the topology of pointwise convergence on $Y$. If $\mathfrak{X}_{\mathfrak{S}}$ is equipped with the topology of pointwise convergence on $\mathfrak{S}$, then $\tau_{T, Y}^{(m)}$ is still stronger than the topology of uniform convergence on compact subsets of $Y$ (see footnote 2). In turn, if there exist integers $i, j, k, l \geqslant 0$ such that $i+j \neq k+l$ and

$$
s^{* i} s^{j}=s^{* k} s^{l}, \quad s \in T,
$$

then $\sup _{\chi \in \mathfrak{X}_{\mathfrak{S}}}|\chi(s)| \leqslant 1$ for every $s \in T$. Hence, in this specific situation, the topology $\tau_{T, Y}^{(m)}$ describes exactly the uniform convergence on $Y$. Property (9) holds for any $T$ which is a unital commutative inverse semigroup (take $i=l=1, j=2$ and $k=0$; cf. [18]); in particular, this is the case for $T$ being an abelian group with involution $s^{*}=s^{-1}$.

Below we stick to the notation declared at the beginning of this section.

Lemma 8. $\mathrm{M}_{\mathrm{f}}^{m}\left(\mathscr{P}_{T}(Y)\right) \subset \mathrm{M}_{+}^{m}\left(\mathscr{P}_{T}(Y)\right) \subset{\overline{\mathrm{M}_{\mathrm{f}}^{m}\left(\mathscr{P}_{\llbracket T \rrbracket}(Y)\right)}}^{\tau} ; \tau=\tau_{\llbracket T \rrbracket, Y}^{(m)}$.

Proof. Only the second inclusion has to be justified. Consider a $\llbracket T \rrbracket$-bounded subset $Z$ of $Y$ and take $w=\left[w_{k, l}\right]_{k, l=1}^{m} \in \mathrm{M}_{+}^{m}\left(\mathscr{P}_{T}(Y)\right)$. By Lemma 7 , the transpose $v$ of $w$ is a member of $\mathrm{M}_{+}^{m}\left(\mathscr{P}_{T^{*}}(Y)\right)$. Note also that $\|v(\chi)\|=\|w(\chi)\|$ for every $\chi \in Y$, and consequently, $M \stackrel{\text { def }}{=} \sup _{\chi \in Z}\|v(\chi)\|<\infty$. Without loss of generality we can assume that $M>0$. By the Weierstrass theorem, there exists a sequence of real polynomials $\left\{\rho_{n}\right\}_{n=1}^{\infty}$ vanishing at 0 which tends to the square root function uniformly on $[0, M]$. For $\chi \in Y$, we denote by $\sqrt{v(\chi)}$ the square root of $v(\chi)$. As for every $\chi \in Z$ the norm of the nonnegative matrix $v(\chi)$ is less than or equal to $M$, we get

$$
\sup _{\chi \in Z}\left\|\sqrt{v(\chi)}-\rho_{n}(v(\chi))\right\| \leqslant \sup _{x \in[0, M]}\left|\sqrt{x}-\rho_{n}(x)\right|, \quad n \geqslant 1 .
$$

Let $\left\{e_{k}\right\}_{k=1}^{m}$ be the standard ' $0-1$ ' basis of $\mathbb{C}^{m}$. Since $\rho_{n}(0)=0$, we see that $\rho_{n}(v(\cdot)) e_{k} \in \mathscr{P}_{\left[T^{*}\right]}\left(Y, \ell^{2}\right)$ (after a natural embedding of $\mathbb{C}^{m}$ into $\ell^{2}$ ) and hence

${ }^{2}$ Note that in view of Tychonoff's theorem $\mathfrak{S}$-bounded sets coincide with subsets of $\mathfrak{X}_{\mathfrak{S}}$ which are relatively compact in the topology of pointwise convergence on $\mathfrak{S}$. 
$\left[\left\langle\rho_{n}(v(\cdot)) e_{k}, \rho_{n}(v(\cdot)) e_{l}\right\rangle_{\ell^{2}}\right]_{k, l=1}^{m} \in \mathrm{M}_{\mathrm{f}}^{m}\left(\mathscr{P}_{\llbracket T \rrbracket}(Y)\right)$. It follows from (10) that the sequence $\left[\left\langle\rho_{n}(v(\cdot)) e_{k}, \rho_{n}(v(\cdot)) e_{l}\right\rangle_{\ell^{2}}\right]_{k, l=1}^{m}$ converges uniformly on $Z$ to $\tilde{v}=\left[\tilde{v}_{k, l}\right]_{k, l=1}^{m}$ as $n \rightarrow \infty$, where $\tilde{v}_{k, l}(\chi) \stackrel{\text { def }}{=}\left\langle\sqrt{v(\chi)} e_{k}, \sqrt{v(\chi)} e_{l}\right\rangle_{\ell^{2}}$ for $\chi \in Y$. Since

$$
\tilde{v}_{k, l}(\chi)=\left\langle v(\chi) e_{k}, e_{l}\right\rangle_{\ell^{2}}=v_{l, k}(\chi)=w_{k, l}(\chi), \quad \chi \in Y, k, l=1, \ldots, m,
$$

and the class of $\llbracket T \rrbracket$-bounded subsets of $Y$ is directed upwards by inclusion, the proof is complete.

Remark 9. One can infer from the proof of Lemma 8 that every matrix in $\mathrm{M}_{+}^{m}\left(\mathscr{P}_{T}(Y)\right)$ can be approximated in the topology $\tau_{\llbracket T \rrbracket, Y}^{(m)}$ by means of matrices of the form $\left[\left\langle p_{k}(\cdot), p_{l}(\cdot)\right\rangle_{\ell^{2}}\right]_{k, l=1}^{m} \in \mathrm{M}_{\mathrm{f}}^{m}\left(\mathscr{P}_{\llbracket T \rrbracket}(Y)\right)$, where $p_{1}, \ldots, p_{m} \in \mathscr{P}_{\left[T^{*}\right]}\left(Y, \ell^{2}\right)$.

Let $\mathcal{D}$ be a complex linear space. Denote by $\mathcal{S}(\mathcal{D})$ the set of all sesquilinear forms on $\mathcal{D}$. For every integer $m \geqslant 1$, we define

$$
\begin{aligned}
& \mathrm{M}^{m}(\mathcal{S}(\mathcal{D}))=\left\{\left[\phi_{k, l}\right]_{k, l=1}^{m}: \phi_{k, l} \in \mathcal{S}(\mathcal{D}) \text { for all } k, l=1, \ldots, m\right\}, \\
& \mathrm{M}_{+}^{m}(\mathcal{S}(\mathcal{D}))=\left\{\left[\phi_{k, l}\right]_{k, l=1}^{m} \in \mathrm{M}^{m}(\mathcal{S}(\mathcal{D})):\left[\phi_{k, l}\right]_{k, l=1}^{m} \gg 0\right\},
\end{aligned}
$$

where the notation $\left[\phi_{k, l}\right]_{k, l=1}^{m} \gg 0$ means that

$$
\sum_{k, l=1}^{m} \phi_{k, l}\left(h_{k}, h_{l}\right) \geqslant 0 \quad \text { for all } h_{1}, \ldots, h_{m} \in \mathcal{D} .
$$

We say that a mapping $\Psi: \mathfrak{S} \rightarrow \mathcal{S}(\mathcal{D})$ is positive definite if

$$
\sum_{k, l=1}^{m} \Psi\left(s_{l}^{*} s_{k}\right)\left(h_{k}, h_{l}\right) \geqslant 0
$$

for every integer $m \geqslant 1$ and for all $s_{1}, \ldots, s_{m} \in \mathfrak{S}$ and $h_{1}, \ldots, h_{m} \in \mathcal{D}$.

Suppose that $Y$ is a determining subset of $\mathfrak{X}_{\mathfrak{S}}$. Then for every mapping $\Phi: T \rightarrow$ $\mathcal{S}(\mathcal{D})$, there exists a unique linear mapping $\Lambda_{\Phi, Y}: \mathscr{P}_{T}(Y) \rightarrow \mathcal{S}(\mathcal{D})$ such that

$$
\Lambda_{\Phi, Y}\left(\left.\hat{s}\right|_{Y}\right)=\Phi(s), \quad s \in T .
$$

We say that the mapping $\Lambda_{\Phi, Y}$ is completely positive if for every integer $m \geqslant 1$,

$$
\Lambda_{\Phi, Y}^{(m)}\left(\mathrm{M}_{+}^{m}\left(\mathscr{P}_{T}(Y)\right)\right) \subset \mathrm{M}_{+}^{m}(\mathcal{S}(\mathcal{D})),
$$

where $\Lambda_{\Phi, Y}^{(m)}: \mathrm{M}^{m}\left(\mathscr{P}_{T}(Y)\right) \rightarrow \mathrm{M}^{m}(\mathcal{S}(\mathcal{D}))$ is a linear mapping defined by

$$
\Lambda_{\Phi, Y}^{(m)}\left(\left[w_{k, l}\right]_{k, l=1}^{m}\right)=\left[\Lambda_{\Phi, Y}\left(w_{k, l}\right)\right]_{k, l=1}^{m}, \quad\left[w_{k, l}\right]_{k, l=1}^{m} \in \mathrm{M}^{m}\left(\mathscr{P}_{T}(Y)\right) .
$$

$\Lambda_{\Phi, Y}$ is said to be completely f-positive if for every integer $m \geqslant 1$,

$$
\Lambda_{\Phi, Y}^{(m)}\left(\mathrm{M}_{\mathrm{f}}^{m}\left(\mathscr{P}_{T}(Y)\right)\right) \subset \mathrm{M}_{+}^{m}(\mathcal{S}(\mathcal{D})) .
$$

Apparently, complete positivity implies complete f-positivity. If $Y=\mathfrak{X}_{\mathfrak{S}}$, we shall abbreviate $\Lambda_{\Phi, Y}\left(\Lambda_{\Phi, Y}^{(m)}\right.$ respectively) to $\Lambda_{\Phi}\left(\Lambda_{\Phi}^{(m)}\right.$ respectively $)$.

We now show that the notion of complete f-positivity of $\Lambda_{\Phi, Y}$ does not depend on the choice of determining set $Y$.

Proposition 10. Suppose that $\mathfrak{S}$ is a unital commutative $*$-semigroup and $T$ is a nonempty subset of $\mathfrak{S}$. Let $\mathcal{D}$ be a complex linear space and $\Phi: T \rightarrow \mathcal{S}(\mathcal{D})$ be a mapping. If $Y$ and $Z$ are determining subsets of $\mathfrak{X}_{\mathfrak{S}}$, then $\Lambda_{\Phi, Y}$ is completely $f$-positive if and only if $\Lambda_{\Phi, Z}$ is completely f-positive. 
Proof. The proof reduces to the case $Z=\mathfrak{X}_{\mathfrak{S}}$. By (7) and (11) we have

$$
\Lambda_{\Phi, Y}^{(m)} \circ \pi_{T, Y}^{(m)}=\Lambda_{\Phi}^{(m)}, \quad m=1,2, \ldots,
$$

which, together with (8), implies the desired equivalence.

For a complex linear space $\mathcal{D}$, we denote by $\varrho_{\mathcal{D}}$ the locally convex topology on $\mathcal{S}(\mathcal{D})$ given by the system of seminorms $\phi \mapsto|\phi(f, g)|$ indexed by all the pairs $(f, g) \in \mathcal{D} \times \mathcal{D}$. Clearly, the topology $\varrho_{\mathcal{D}}$ is nothing other than that of pointwise convergence on $\mathcal{D} \times \mathcal{D}$, and therefore it can be regarded as an analogue of the weak operator topology on the set of bounded linear operators on a Hilbert space.

Proposition 11. Assume that $\mathfrak{S}$ is a unital commutative $*$-semigroup, $T$ is a nonempty subset of $\mathfrak{S}$ such that $\llbracket T \rrbracket \subset T$, and $Y$ is a determining subset of $\mathfrak{X}_{\mathfrak{S}}$. Let $\mathcal{D}$ be a complex linear space and $\Phi: T \rightarrow \mathcal{S}(\mathcal{D})$ be a mapping. Suppose that the mapping $\Lambda_{\Phi, Y}:\left(\mathscr{P}_{T}(Y), \tau_{T, Y}\right) \rightarrow\left(\mathcal{S}(\mathcal{D}), \varrho_{\mathcal{D}}\right)$ is continuous. Then $\Lambda_{\Phi, Y}$ is completely f-positive if and only if $\Lambda_{\Phi, Y}$ is completely positive.

Proof. One can check that the assumed continuity of $\Lambda_{\Phi, Y}$ implies that of

$$
\Lambda_{\Phi, Y}^{(m)}:\left(\mathrm{M}^{m}\left(\mathscr{P}_{T}(Y)\right), \tau_{T, Y}^{(m)}\right) \rightarrow\left(\mathrm{M}^{m}(\mathcal{S}(\mathcal{D})), \varrho_{\mathcal{D}}^{(m)}\right), \quad m=1,2, \ldots
$$

Hence, by the $\varrho_{\mathcal{D}}^{(m)}$-closedness of $\mathrm{M}_{+}^{m}(\mathcal{S}(\mathcal{D}))$ and Lemma 8 , we arrive at the desired conclusion.

Regarding Proposition 11, note that if $T$ is a $*$-subsemigroup of a unital commutative $*$-semigroup $\mathfrak{S}$ ( $T$ need not be unital), then $\llbracket T \rrbracket \subset T$. The reverse implication is not true in general. In fact, it may happen that $\llbracket T \rrbracket \subset T$ although $T$ is not a subsemigroup of $\mathfrak{S}$ and $T \neq T^{*}$ (thus neither $T \subset \llbracket T \rrbracket$ nor $T^{*} \subset \llbracket T \rrbracket$ holds). We leave it to the reader to verify that this is the case for the subset

$$
T \stackrel{\text { def }}{=}\{(k, l) \in \mathfrak{N}: k \geqslant 1, l \geqslant 1\} \cup T_{0}
$$

of the $*$-semigroup $\mathfrak{N}$ considered in Example 3 , where $T_{0}$ is a proper subset of $\{(k, 0) \in \mathfrak{N}: k \geqslant 1\}$ containing $(1,0)$.

4. Criteria for extendibility. As mentioned in the Introduction, not every positive definite function on $\mathfrak{N}$ extends to a positive definite function on the $*$-semigroup $\mathfrak{N}_{+}$(see Example 3 and Section 5 for the definitions). However, if we impose a stronger positivity-like condition on a function defined on $\mathfrak{N}$ (in the language of [10] this is positive definiteness with respect to $\mathfrak{N}_{+}$), then it is extendable to a positive definite function on $\mathfrak{N}_{+}$, and reversely (cf. [54]). The property of extendibility of positive definite functions was characterized likewise in [54, also in the case of *-subsemigroups of abstract (unital commutative) *-semigroups. This led the authors of [54 to find a new solution of the complex moment problem (not to mention other extension results). The key feature that made this approach successful was the semiperfectness of $\mathfrak{N}_{+}$, the property guaranteeing that every positive definite function on $\mathfrak{N}_{+}$is a moment function, i.e. it has the Laplace-type integral representation on the dual $*$-semigroup of $\mathfrak{N}_{+}$. Inspired by this, Bisgaard attached to any $*$-semigroup $\mathfrak{S}$ an enveloping perfect ${ }^{3} *$-semigroup $\mathfrak{Q}$ such that the set of all moment functions on $\mathfrak{S}$ coincides with the set of all functions which are positive definite with respect to $\mathfrak{Q}$ (cf. [10]). The instance of a semiperfect (but not perfect) *-semigroup $\mathfrak{N}_{+}$as an extending $*$-semigroup for $\mathfrak{N}$ shows that semiperfectness is

\footnotetext{
${ }^{3}$ I.e. semiperfect with the uniqueness of integral representation.
} 
sufficient as far as moment problems are concerned. Though there is a limited freedom of choice of an extending semiperfect *-semigroup for a fixed $*$-semigroup, it can by no means be chosen arbitrarily, as indicated in the discussion concerning the inclusions (22) in 54. In this section we are looking for criteria that guarantee positive definite extendibility of mappings defined on symmetric subsets of (operator) semiperfect $*$-semigroups. As a result, we obtain the characterizations of "truncated" moment functions enriching those in [54, 55] with the new positivity conditions of the Riesz-Haviland type.

Suppose that $\mathrm{M}$ is a $\sigma$-algebra of subsets of a set $X \neq \varnothing$ and $\mathcal{D}$ is a complex linear space. A mapping $\mu: \mathrm{M} \rightarrow \mathcal{S}(\mathcal{D})$ is called a semispectral measure 4 on $\mathrm{M}$ if $\mu(\cdot)(f, f)$ is a finite positive measure for every $f \in \mathcal{D}$.

Let $\mathfrak{S}$ be a unital commutative *-semigroup. Denote by $\mathrm{M}_{\mathfrak{S}}$ the smallest $\sigma$ algebra of subsets of $\mathfrak{X}_{\mathfrak{S}}$ with respect to which all the transforms $\hat{s}, s \in \mathfrak{S}$, are measurable. Following [7, we say that $\mathfrak{S}$ is operator semiperfect if for any complex linear space $\mathcal{D}$ and for any positive definite mapping $\Psi: \mathfrak{S} \rightarrow \mathcal{S}(\mathcal{D})$ there exists a semispectral measure $\mu: \mathrm{M}_{\mathfrak{S}} \rightarrow \mathcal{S}(\mathcal{D})$ such that

$$
\Psi(s)=\int_{\mathfrak{X}_{\mathfrak{S}}} \hat{s}(\chi) \mu(\mathrm{d} \chi), \quad s \in \mathfrak{S} .
$$

This equality is to be understood in the following sense:

$$
\Psi(s)(f, g)=\int_{\mathfrak{X}_{\mathfrak{S}}} \hat{s}(\chi) \mu(\mathrm{d} \chi)(f, g), \quad f, g \in \mathcal{D}, s \in \mathfrak{S} ;
$$

here and henceforth all the integrands are tacitly assumed to be summable. An equivalent definition of operator semiperfectness may be stated in a matrix-type form, as shown in 7].

Note that if $\mathfrak{X}_{\mathfrak{S}}$ is equipped with the topology of pointwise convergence on $\mathfrak{S}$, then the transforms $\hat{s}, s \in \mathfrak{S}$, are continuous, and consequently the $\sigma$-algebra $\mathrm{M}_{\mathfrak{S}}$ is contained in the $\sigma$-algebra of all Borel subsets of $\mathfrak{X}_{\mathfrak{S}}$ (the equality does not hold in general). It is also clear that if $\mathrm{M}$ is a $\sigma$-algebra of subsets of $\mathfrak{X}_{\mathfrak{S}}$ such that $\mathrm{M}_{\mathfrak{S}} \subset \mathrm{M}$ and $\mu$ is a semispectral measure on $\mathrm{M}$ satisfying (12), then the restriction of $\mu$ to $M_{\mathfrak{S}}$ satisfies (12) as well.

Lemma 12. Let $\mathfrak{S}$ be a unital commutative *-semigroup whose dual $*$-semigroup $\mathfrak{X}_{\mathfrak{S}}$ is determining and let $T$ be a nonempty subset of $\mathfrak{S}$. Suppose that a complex linear space $\mathcal{D}$ and a mapping $\Phi: T \rightarrow \mathcal{S}(\mathcal{D})$ are given.

(i) If $\mu: \mathrm{M} \rightarrow \mathcal{S}(\mathcal{D})$ is a semispectral measure on a $\sigma$-algebra $\mathrm{M}$ of subsets of $\mathfrak{X}_{\mathfrak{S}}, \mathrm{M}_{\mathfrak{S}} \subset \mathrm{M}$ and $Y$ is a determining subset of $\mathfrak{X}_{\mathfrak{S}}$ such that $Y \in \mathrm{M}$ and

$$
\Phi(s)=\int_{Y} \hat{s} \mathrm{~d} \mu, \quad s \in T,
$$

then $\Lambda_{\Phi, Y}$ is completely positive.

(ii) If $T=\mathfrak{S}$ and $\Lambda_{\Phi}$ is completely f-positive, then $\Phi$ is positive definite.

In particular, if $\mathfrak{S}$ is operator semiperfect and $T=\mathfrak{S}$, then $\Phi$ is positive definite if and only if $\Lambda_{\Phi}$ is completely f-positive or equivalently if $\Lambda_{\Phi}$ is completely positive.

Proof. (i) Take $\left[w_{k, l}\right]_{k, l=1}^{m} \in \mathrm{M}_{+}^{m}\left(\mathscr{P}_{T}(Y)\right)$ and $e_{1}, \ldots, e_{m} \in \mathcal{D}$. Notice that the complex measures $\mu(\cdot)\left(e_{i}, e_{j}\right), i, j \in\{1, \ldots, m\}$, are absolutely continuous with

\footnotetext{
${ }^{4}$ With the natural identification of bounded linear operators with sesquilinear forms, our definition subsumes the classical semispectral operator-valued measures (cf. [38]).
} 
respect to some finite positive measure $\nu$ on $\mathrm{M}\left(\right.$ e.g. $\left.\nu=\sum_{i=1}^{m} \mu(\cdot)\left(e_{i}, e_{i}\right)\right)$. By the Radon-Nikodym theorem, there exists a system $\left\{h_{i, j}\right\}_{i, j=1}^{m}$ of M-measurable complex functions on $\mathfrak{X}_{\mathfrak{S}}$ such that $\mu(\sigma)\left(e_{i}, e_{j}\right)=\int_{\sigma} h_{i, j} \mathrm{~d} \nu$ for all $\sigma \in \mathrm{M}$. Since $\left[\mu(\sigma)\left(e_{i}, e_{j}\right)\right]_{i, j=1}^{m} \geqslant 0$ for every $\sigma \in \mathrm{M}$, one can show (see the proof of [38, Theorem 6.4]) that there exists $Z \in \mathrm{M}$ such that $\nu\left(\mathfrak{X}_{\mathfrak{S}} \backslash Z\right)=0$ and $\left[h_{k, l}(\chi)\right]_{k, l=1}^{m} \geqslant 0$ for every $\chi \in Z$. Let for $\chi \in Z,\left[a_{k, l}(\chi)\right]_{k, l=1}^{m}$ be the square root of $\left[h_{k, l}(\chi)\right]_{k, l=1}^{m}$. Since $\left[w_{k, l}(\chi)\right]_{k, l=1}^{m} \geqslant 0$ for every $\chi \in Y$ and $\nu(Y \backslash(Y \cap Z))=0$, we get

$$
\sum_{k, l=1}^{m} \Lambda_{\Phi, Y}\left(w_{k, l}\right)\left(e_{k}, e_{l}\right)=\int_{Y \cap Z} \sum_{k, l=1}^{m} w_{k, l} h_{k, l} \mathrm{~d} \nu=\int_{Y \cap Z} \sum_{j=1}^{m} \sum_{k, l=1}^{m} w_{k, l} a_{k, j} \overline{a_{l, j}} \mathrm{~d} \nu \geqslant 0 .
$$

(ii) Take finite sequences $s_{1}, \ldots, s_{m} \in \mathfrak{S}$ and $e_{1}, \ldots, e_{m} \in \mathcal{D}$. It is easily seen that $\left[\widehat{s}_{k} s_{l}^{*}\right]_{k, l=1}^{m} \in \mathrm{M}_{\mathrm{f}}^{m}\left(\mathscr{P}\left(\mathfrak{X}_{\mathfrak{S}}\right)\right)$. By complete f-positivity of $\Lambda_{\Phi}$, we get $\sum_{k, l=1}^{m} \Phi\left(s_{l}^{*} s_{k}\right)\left(e_{k}, e_{l}\right) \geqslant 0$, which finishes the proof.

We now deal with the question of when a $\mathcal{S}(\mathcal{D})$-valued mapping extends from a subset $T$ of $\mathfrak{S}$ to a positive definite mapping on the whole of $\mathfrak{S}$. The following result is the main tool in our considerations. It is proved as a consequence of Theorem 1 of [55] (in fact, a prototype of this theorem appeared in [54]). Below, we interpret the algebraic tensor product $\mathcal{F}_{\mathfrak{S}} \otimes \ell^{2}$ as the collection of all $\ell^{2}$-valued functions on $\mathfrak{S}$ vanishing off finite sets. Revoking the definition of $\mathfrak{S}_{\mathfrak{h}}$ from page 547 , it may be convenient for further references to detach the following condition:

$$
T \text { is a symmetric subset of } \mathfrak{S} \text { containing } \mathfrak{S}_{\mathfrak{h}} \text {. }
$$

Lemma 13. Suppose that $\mathfrak{S}$ is a unital commutative $*$-semigroup, $T$ satisfies (13) and $Y$ is a determining subset of $\mathfrak{X}_{\mathfrak{S}}$. If $\mathcal{D}$ is a complex linear space, then for every mapping $\Phi: T \rightarrow \mathcal{S}(\mathcal{D})$ the following three conditions are equivalent:

(i) $\Phi$ extends to a positive definite mapping $\Psi: \mathfrak{S} \rightarrow \mathcal{S}(\mathcal{D})$,

(ii) $\sum_{k, l=1}^{m} \sum_{\substack{s, t \in \mathfrak{S} \\ t^{*} s \in T}} \Phi\left(t^{*} s\right)\left(e_{k}, e_{l}\right)\left\langle\lambda_{k}(s), \lambda_{l}(t)\right\rangle_{\ell^{2}} \geqslant 0$ for every integer $m \geqslant 1$ and for all finite systems $\left\{e_{n}\right\}_{n=1}^{m} \subset \mathcal{D}$ and $\left\{\lambda_{n}\right\}_{n=1}^{m} \subset \mathcal{F}_{\mathfrak{S}} \otimes \ell^{2}$ such that

$$
\sum_{\substack{s, t \in \mathfrak{S} \\ t^{*} s=u}}\left\langle\lambda_{i}(s), \lambda_{j}(t)\right\rangle_{\ell^{2}}=0, \quad u \in \mathfrak{S} \backslash T, i, j=1, \ldots, m
$$

(iii) $\Lambda_{\Phi, Y}$ is completely f-positive.

Proof. (i) $\Leftrightarrow$ (ii) The reader can convince himself that condition (ii) of [55, Theorem 1] is equivalent to our condition (ii); note that this can be shown directly, without recourse to the proof given therein. Hence, our equivalence (i) $\Leftrightarrow$ (ii) is a consequence of [55, Theorem 1].

(ii) $\Rightarrow$ (iii) Fix an integer $m \geqslant 1$. Take $w=\left[\left\langle p_{k}(\cdot), p_{l}(\cdot)\right\rangle_{\ell^{2}}\right]_{k, l=1}^{m} \in \mathrm{M}_{\mathrm{f}}^{m}\left(\mathscr{P}_{T}(Y)\right)$ with $p_{1}, \ldots, p_{m} \in \mathscr{P}\left(Y, \ell^{2}\right)$. Then there exist $\lambda_{1}, \ldots, \lambda_{m} \in \mathcal{F}_{\mathfrak{S}} \otimes \ell^{2}$ such that

$$
p_{k}(\chi)=\sum_{s \in \mathfrak{S}} \chi(s) \lambda_{k}(s), \quad \chi \in Y, k=1, \ldots, m .
$$

It is clear that

$$
\left\langle p_{k}(\chi), p_{l}(\chi)\right\rangle_{\ell^{2}}=\sum_{u \in \mathfrak{S}} \chi(u) \sum_{\substack{s, t \in \mathfrak{S} \\ t^{*} s=u}}\left\langle\lambda_{k}(s), \lambda_{l}(t)\right\rangle_{\ell^{2}}, \quad \chi \in Y, k, l=1, \ldots, m .
$$


Since $w \in \mathrm{M}^{m}\left(\mathscr{P}_{T}(Y)\right)$ and $Y$ is determining, we see that (16) implies (14). It follows from (11), (14), (16) and (ii) that

$$
\begin{aligned}
\sum_{k, l=1}^{m} \Lambda_{\Phi, Y}\left(\left\langle p_{k}(\cdot), p_{l}(\cdot)\right\rangle_{\ell^{2}}\right)\left(e_{k}, e_{l}\right) & =\sum_{k, l=1}^{m} \sum_{u \in T} \sum_{\substack{s, t \in \mathfrak{S} \\
t^{*} s=u}} \Phi(u)\left(e_{k}, e_{l}\right)\left\langle\lambda_{k}(s), \lambda_{l}(t)\right\rangle_{\ell^{2}} \\
& =\sum_{k, l=1}^{m} \sum_{\substack{s, t \in \mathfrak{S} \\
t^{*} s \in T}} \Phi\left(t^{*} s\right)\left(e_{k}, e_{l}\right)\left\langle\lambda_{k}(s), \lambda_{l}(t)\right\rangle_{\ell^{2}} \geqslant 0
\end{aligned}
$$

for all vectors $e_{1}, \ldots, e_{m} \in \mathcal{D}$. This shows that $\Lambda_{\Phi, Y}$ is completely f-positive.

Reversing the above reasoning, we infer (ii) from (iii); to see this, for fixed $\lambda_{1}, \ldots, \lambda_{m} \in \mathcal{F}_{\mathfrak{S}} \otimes \ell^{2}$ consider $p_{1}, \ldots, p_{m} \in \mathscr{P}\left(Y, \ell^{2}\right)$ defined by (15). This completes the proof.

With the above discussions, we are in a position to state the main result of the paper which supplies criteria for extendibility to a positive definite function.

Theorem 14. Suppose that $\mathfrak{S}$ is an operator semiperfect $*$-semigroup, $T$ satisfies (13) and $Y$ is a determining subset of $\mathfrak{X}_{\mathfrak{S}}$. If $\mathcal{D}$ is a complex linear space, then for every mapping $\Phi: T \rightarrow \mathcal{S}(\mathcal{D})$ the following four conditions are equivalent:

(i) $\Phi$ extends to a positive definite mapping $\Psi: \mathfrak{S} \rightarrow \mathcal{S}(\mathcal{D})$,

(ii) $\Phi(s)=\int_{\mathfrak{X}_{\mathfrak{S}}} \hat{s} \mathrm{~d} \mu$ for all $s \in T$ with some semispectral measure $\mu$ on $\mathrm{M}_{\mathfrak{S}}$,

(iii) $\Lambda_{\Phi}$ is completely positive 5

(iv) $\Lambda_{\Phi, Y}$ is completely f-positive.

Proof. (i) $\Rightarrow$ (ii) Use operator semiperfectness of $\mathfrak{S}$.

(ii) $\Rightarrow$ (i) Since $|\hat{s}|^{2}=\widehat{s^{*} s}$ and $s^{*} s \in T$ for every $s \in \mathfrak{S}$, we see that the function $\hat{s}$ is square summable with respect to $\mu$ for every $s \in \mathfrak{S}$. This enables us to define the mapping $\Psi: \mathfrak{S} \rightarrow \mathcal{S}(\mathcal{D})$ by

$$
\Psi(s)=\int_{\mathfrak{X}_{\mathfrak{S}}} \hat{s} \mathrm{~d} \mu, \quad s \in \mathfrak{S} .
$$

It follows from Lemma 12 that $\Psi$ is a positive definite extension of $\Phi$.

(i) $\Leftrightarrow$ (iv) Apply Lemma 13 ,

(ii) $\Rightarrow$ (iii) This is a consequence of Lemma 12 .

(iii) $\Rightarrow$ (iv) Since (iii) implies (iv) with $Y=\mathfrak{X}_{\mathfrak{S}}$, we see that $\Lambda_{\Phi}$ is completely f-positive. An application of Proposition 10 guarantees that $\Lambda_{\Phi, Y}$ is completely f-positive as well. This completes the proof.

We now turn to the the case of scalar functions. A unital commutative *semigroup $\mathfrak{S}$ is called semiperfect (cf. [7]) if for any positive definite function $\Psi: \mathfrak{S} \rightarrow \mathbb{C}$ there exists a finite positive measure $\mu$ on $\mathrm{M}_{\mathfrak{S}}$ such that

$$
\Psi(s)=\int_{\mathfrak{X}_{\mathfrak{S}}} \hat{s}(\chi) \mu(\mathrm{d} \chi), \quad s \in \mathfrak{S} .
$$

Evidently, operator semiperfectness implies semiperfectness, but not conversely as indicated by Bisgaard in [11. The following is a scalar counterpart of Theorem 14.

${ }^{5}$ It follows from our assumptions that $\mathfrak{X}_{\mathfrak{S}}$ is determining, which makes it legitimate to consider $\Lambda_{\Phi}$. 
Theorem 15. Suppose that $\mathfrak{S}$ is a semiperfect $*$-semigroup, $T$ satisfies (13) and $Y$ is a determining subset of $\mathfrak{X}_{\mathfrak{S}}$. Then for every function $\varphi: T \rightarrow \mathbb{C}$ the following four conditions are equivalent:

(i) $\varphi$ extends to a positive definite function $\psi: \mathfrak{S} \rightarrow \mathbb{C}$,

(ii) $\varphi(s)=\int_{\mathfrak{X}_{\mathfrak{S}}} \hat{s} \mathrm{~d} \mu$ for all $s \in T$ with some positive measure $\mu$ on $\mathrm{M}_{\mathfrak{S}}$,

(iii) $\Lambda_{\varphi}(p) \geqslant 0$ for every $p \in \mathscr{P}_{T}\left(\mathfrak{X}_{\mathfrak{S}}\right)$ such that $p(\chi) \geqslant 0$ for all $\chi \in \mathfrak{X}_{\mathfrak{S}}$,

(iv) $\Lambda_{\varphi, Y}(p) \geqslant 0$ for every $p \in \mathscr{P}_{T}(Y)$ for which there exist finitely many functions $q_{1}, \ldots, q_{n} \in \mathscr{P}(Y)$ such that $p(\chi)=\sum_{j=1}^{n}\left|q_{j}(\chi)\right|^{2}, \chi \in Y$.

Proof. Note first that the functional $\Lambda_{\varphi}$ is completely positive if and only if (iii) holds. Indeed, if (iii) holds and $w=\left[w_{i, j}\right]_{i, j=1}^{m} \in \mathrm{M}_{+}^{m}\left(\mathscr{P}_{T}\left(\mathfrak{X}_{\mathfrak{S}}\right)\right)$, then for every $\lambda=$ $\left(\lambda_{1}, \ldots, \lambda_{m}\right) \in \mathbb{C}^{m}$, the function $p_{\lambda} \stackrel{\text { def }}{=} \sum_{i, j=1}^{m} \lambda_{i} \overline{\lambda_{j}} w_{i, j} \in \mathscr{P}_{T}\left(\mathfrak{X}_{\mathfrak{S}}\right)$ is nonnegative on $\mathfrak{X}_{\mathfrak{S}}$, and consequently

$$
\sum_{i, j=1}^{m} \lambda_{i} \overline{\lambda_{j}} \Lambda_{\varphi}\left(w_{i, j}\right)=\Lambda_{\varphi}\left(p_{\lambda}\right) \geqslant 0 .
$$

This means that $\left[\Lambda_{\varphi}\left(w_{i, j}\right)\right]_{i, j=1}^{m} \geqslant 0$. The reverse implication is obvious.

The next observation is that the functional $\Lambda_{\varphi, Y}$ is completely f-positive if and only if (iv) holds. Indeed, if (iv) holds and $w=\left[\left\langle p_{k}(\cdot), p_{l}(\cdot)\right\rangle_{\ell^{2}}\right]_{k, l=1}^{m} \in \mathrm{M}_{\mathrm{f}}^{m}\left(\mathscr{P}_{T}(Y)\right)$ with some $p_{1}, \ldots, p_{m} \in \mathscr{P}\left(Y, \ell^{2}\right)$, then for every $\lambda=\left(\lambda_{1}, \ldots, \lambda_{m}\right) \in \mathbb{C}^{m}$ the function $q_{\lambda} \stackrel{\text { def }}{=} \sum_{i=1}^{m} \lambda_{i} p_{i}$ belongs to $\mathscr{P}\left(Y, \ell^{2}\right)$, whereas $\left\langle q_{\lambda}(\cdot), q_{\lambda}(\cdot)\right\rangle_{\ell^{2}}$ is in $\mathscr{P}_{T}(Y)$. Hence, by Lemma 6] we have

$$
\sum_{i, j=1}^{m} \lambda_{i} \overline{\lambda_{j}} \Lambda_{\varphi, Y}\left(\left\langle p_{i}(\cdot), p_{j}(\cdot)\right\rangle_{\ell^{2}}\right)=\Lambda_{\varphi, Y}\left(\left\langle q_{\lambda}(\cdot), q_{\lambda}(\cdot)\right\rangle_{\ell^{2}}\right) \geqslant 0
$$

which means that $\left[\Lambda_{\varphi, Y}\left(\left\langle p_{i}(\cdot), p_{j}(\cdot)\right\rangle_{\ell^{2}}\right)\right]_{i, j=1}^{m} \geqslant 0$. The reverse implication is plain.

The above enables us to adapt the proof of Theorem 14 to the present context.

\section{Applications}

5. The truncated complex moment problem. The $*$-semigroup $\mathfrak{N}_{+}$we intend to investigate comes from [54. It plays a crucial role in the complex moment problem. The initial part of this section is devoted to a description of $\mathscr{P}_{T}(Y)$ in this particular case.

Denote by $\mathfrak{N}_{+}$the $*$-semigroup $(\{(m, n) \in \mathbb{Z} \times \mathbb{Z}: m+n \geqslant 0\},+, *)$ with coordinatewise defined addition as a semigroup operation and involution $(m, n)^{*}=(n, m)$. Owing to [54, Remark 7], the dual $*$-semigroup $\mathfrak{X}_{\mathfrak{N}_{+}}$can be identified algebraically with the $*$-subsemigroup $(\Omega \cup(\{0\} \times \mathbb{T}), \cdot, *)$ of the product $*$-semigroup $(\mathbb{C} \times \mathbb{T}, \cdot, *)$ (with coordinatewise defined multiplication as a semigroup operation and involution $\left.(z, w)^{*}=(\bar{z}, \bar{w})\right)$, where

$$
\Omega \stackrel{\text { def }}{=}\left\{\left(z, z \bar{z}^{-1}\right): z \in \mathbb{C}_{*}\right\} .
$$

Under this identification, $\widehat{(m, n)}$ is given by

$$
\widehat{(m, n)}(z, w)=\chi_{z, w}(m, n)= \begin{cases}z^{m} \bar{z}^{n} & \text { if } z \neq 0 \\ 0 & \text { if } z=0 \text { and } m+n>0 \\ w^{m} & \text { if } z=0 \text { and } m+n=0\end{cases}
$$


for all $(z, w) \in \Omega \cup(\{0\} \times \mathbb{T})$ and $(m, n) \in \mathfrak{N}_{+}$. Given a subset $Z$ of $\mathbb{C}_{*}$, we write

$$
Y_{Z}=\left\{\left(z, z \bar{z}^{-1}\right): z \in Z\right\} \subset \Omega .
$$

By the above identification, we see that if $T$ is a nonempty subset of $\mathfrak{N}_{+}$, then $\mathscr{P}_{T}\left(Y_{Z}\right)$ may be regarded as the set of all rational functions $p$ on $Z$ of the form

$$
p(z, \bar{z})=\sum_{(m, n) \in T} a_{m, n} z^{m} \bar{z}^{n}, \quad z \in Z,
$$

where $\left\{a_{m, n}\right\}_{(m, n) \in T} \subset \mathbb{C}$ is a finite system.

For a subset $T$ of $\mathbb{Z} \times \mathbb{Z}$, we denote by $\mathbb{C}_{T}(z, \bar{z})$ the set of all rational functions $p$ of the form (19) with $Z=\mathbb{C}_{*}$, where $\left\{a_{m, n}\right\}_{(m, n) \in T} \subset \mathbb{C}$ is a finite system. If $T \subset \mathfrak{N}$, where $\mathfrak{N}$ is as in Example 3 , then $\mathbb{C}_{T}(z, \bar{z})$ coincides with the set $\mathbb{C}_{T}[z, \bar{z}]$ of all complex polynomials in $z$ and $\bar{z}$ whose coefficients vanish for all indices in $\mathfrak{N} \backslash T$; hence $\mathbb{C}_{\mathfrak{N}}[z, \bar{z}]=\mathbb{C}[z, \bar{z}]$. It is worth noticing that members of $\mathbb{C}_{\mathfrak{N}_{+}}(z, \bar{z})$ are functions which are bounded on every punctured disc $\{z \in \mathbb{C}: 0<|z| \leqslant R\}, R>0$. This is due to the fact that all terms $z^{m} \bar{z}^{n}$ with $m+n \geqslant 0$ share this property. As a consequence, the set $\mathbb{C}_{\mathfrak{N}_{+}}(z, \bar{z})$ is essentially smaller than $\mathbb{C}_{\mathbb{Z} \times \mathbb{Z}}(z, \bar{z})$. In fact, members of $\mathbb{C}_{\mathfrak{N}_{+}}(z, \bar{z})$ can be characterized by their boundedness on the fixed punctured disc $\{z \in \mathbb{C}: 0<|z| \leqslant 1\}$.

Proposition 16. If $p \in \mathbb{C}_{\mathbb{Z} \times \mathbb{Z}}(z, \bar{z})$, then the following conditions are equivalent:

(i) $p \in \mathbb{C}_{\mathfrak{N}_{+}}(z, \bar{z})$,

(ii) $\sup \{|p(z, \bar{z})|: z \in \mathbb{C}, 0<|z| \leqslant 1\}<\infty$.

Proof. Since the implication (i) $\Rightarrow$ (ii) has been clarified above, we can focus on the implication (ii) $\Rightarrow$ (i). Assume that (ii) holds and $p$ is as in (19) with $T=\mathbb{Z} \times \mathbb{Z}$ and $Z=\mathbb{C}_{*}$. We may write the rational function $p$ in the form $p=p_{0}+\sum_{j=1}^{k} p_{j}$, where $k$ is a positive integer, $p_{0} \in \mathbb{C}_{\mathfrak{N}_{+}}(z, \bar{z})$ and

$$
p_{j}(z, \bar{z})=\sum_{\substack{m, n \in \mathbb{Z} \\ m+n=-j}} a_{m, n} z^{m} \bar{z}^{n}=|z|^{-j} \sum_{\substack{m, n \in \mathbb{Z} \\ m+n=-j}} a_{m, n}\left[\frac{z}{|z|}\right]^{m}\left[\frac{\bar{z}}{|z|}\right]^{n}
$$

for $z \in \mathbb{C}_{*}$ and $j=1, \ldots, k$. Then

$$
|z|^{k-1} p_{k}(z, \bar{z})=|z|^{k-1}\left(p(z, \bar{z})-p_{0}(z, \bar{z})-\sum_{j=1}^{k-1} p_{j}(z, \bar{z})\right), \quad z \in \mathbb{C}_{*},
$$

which, together with (ii) and (20), implies that the function $z \mapsto|z|^{k-1} p_{k}(z, \bar{z})$ is bounded on $\{z \in \mathbb{C}: 0<|z| \leqslant 1\}$. Substituting $z=r \mathrm{e}^{\mathrm{i} \theta}$ with real $\theta$ and $r>0$, and using (20), we deduce that $p_{k}(z, \bar{z})=0$ for all $z \in \mathbb{C}_{*}$. Repeating this argument, we see that $p_{1}(z, \bar{z})=\ldots=p_{k}(z, \bar{z})=0$ for all $z \in \mathbb{C}_{*}$. By Lemma 5 , this completes the proof.

We are now in need of adapting the notion of a determining set (cf. Example 3) to the context of rational functions. Given $T \subset \mathbb{Z} \times \mathbb{Z}$, we say that a subset $Z$ of $\mathbb{C}_{*}$ is determining for $\mathbb{C}_{T}(z, \bar{z})$ (or for short $\mathbb{C}_{T}(z, \bar{z})$-determining) if every rational function $p \in \mathbb{C}_{T}(z, \bar{z})$ vanishing on $Z$ (i.e. $p(z, \bar{z})=0$ for all $z \in Z$ ) vanishes on $\mathbb{C}_{*}$. By Lemma 5 , the set $Z$ is $\mathbb{C}_{T}(z, \bar{z})$-determining if and only if the system of functions $z \mapsto z^{m} \bar{z}^{n},(m, n) \in T$, is linearly independent in $\mathbb{C}^{Z}$. If $T \subset \mathbb{Z}_{+} \times \mathbb{Z}_{+}$, then we allow 0 to be a member of a determining set. Clearly, if $T \subset \mathbb{Z} \times \mathbb{Z}$ and $Z \subset \mathbb{C}_{*}$ is a determining set for $\mathbb{C}_{\mathbb{Z} \times \mathbb{Z}}(z, \bar{z})$, then $Z$ is determining for $\mathbb{C}_{T}(z, \bar{z})$. The 
reverse is not true, e.g. for $T=\left\{(n, n): n \in \mathbb{Z}_{+}\right\}$and $Z=\left\{z \in \mathbb{C}_{*}: z=\bar{z}\right\}$. Lemma 5 provides examples of $\mathbb{C}_{\mathbb{Z} \times \mathbb{Z}}(z, \bar{z})$-determining sets. In particular, each subset $Z$ of $\mathbb{C}_{*}$ with nonempty interior is $\mathbb{C}_{\mathbb{Z} \times \mathbb{Z}}(z, \bar{z})$-determining. Moreover, as shown below, the determining property is preserved when removing a finite subset.

Lemma 17. If $Z$ is a subset of $\mathbb{C}$, then the following conditions are equivalent:

(i) $Z$ is determining for $\mathbb{C}[z, \bar{z}]$,

(ii) $Z \backslash Z_{0}$ is determining for $\mathbb{C}[z, \bar{z}]$ whenever $Z_{0} \subset \mathbb{C}$ is finite,

(iii) $Z \backslash\left(Z_{0} \cup\{0\}\right)$ is determining for $\mathbb{C}_{\mathbb{Z} \times \mathbb{Z}}(z, \bar{z})$ whenever $Z_{0} \subset \mathbb{C}$ is finite.

Proof. (i) $\Rightarrow$ (iii) Suppose that $Z_{0}=\left\{\lambda_{1}, \ldots, \lambda_{k}\right\}$ and take $p \in \mathbb{C}_{\mathbb{Z} \times \mathbb{Z}}(z, \bar{z})$ such that $p(z, \bar{z})=0$ for all $z \in Z \backslash\left(Z_{0} \cup\{0\}\right)$. Then for a sufficiently large positive integer $N$ the rational function $q(z, \bar{z}) \stackrel{\text { def }}{=} z^{N} \bar{z}^{N}\left(z-\lambda_{1}\right) \ldots\left(z-\lambda_{k}\right) p(z, \bar{z})$ is a polynomial in $z$ and $\bar{z}$ such that $q(z, \bar{z})=0$ for all $z \in Z$. By $(\mathrm{i}), q(z, \bar{z})=0$ for all $z \in \mathbb{C}$, and so $p(z, \bar{z})=0$ for all $z \in \mathbb{C} \backslash\left(Z_{0} \cup\{0\}\right)$. As a consequence, $p(z, \bar{z})=0$ for all $z \in \mathbb{C}_{*}$.

Implications (iii) $\Rightarrow($ ii) and (ii) $\Rightarrow($ i) are evident.

The following simple fact is crucial for further investigations. It enables us to deal with the complex plane instead of a larger and less handy dual $*$-semigroup $\mathfrak{X}_{\mathfrak{N}_{+}}$.

Lemma 18. A subset $Z$ of $\mathbb{C}_{*}$ is determining for $\mathbb{C}_{\mathfrak{N}_{+}}(z, \bar{z})$ if and only if the set $Y_{Z}$ defined in (18) is determining for $\mathscr{P}\left(\mathfrak{X}_{\mathfrak{N}_{+}}\right)$.

Proof. Apply Lemma 5 and the description (17) of $\widehat{\mathfrak{N}_{+}}$.

A sequence $\left\{c_{m, n}\right\}_{m, n=0}^{\infty} \subset \mathbb{C}$ is called a complex moment sequence if there exists a positive Borel measure $\mu$ on $\mathbb{C}$ such that

$$
c_{m, n}=\int_{\mathbb{C}} z^{m} \bar{z}^{n} \mu(\mathrm{d} z), \quad m, n \geqslant 0 .
$$

A measure $\mu$ need by no means be unique (for this and related questions we refer the reader to [48] and [21]).

The following result is an extension of Theorem 1 of 54 as well as of the "support-free" complex version of the Riesz-Haviland theorem (see [34]). The first of the two theorems can be seen as the equivalence (i) $\Longleftrightarrow$ (iii) below with $T=\mathbb{Z}_{+} \times \mathbb{Z}_{+}$, while the other as the equivalence (i) $\Longleftrightarrow$ (iv) with the same $T$.

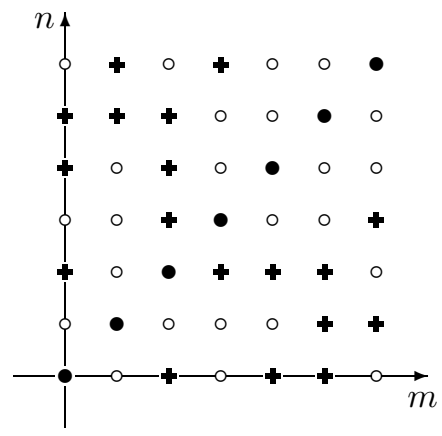

Figure 1. $T$ appearing in Theorem 19- an example: - - obligatory data, + - additional data, o - missing data. 
Theorem 19. Let $T$ be a symmetric subset of $\mathbb{Z}_{+} \times \mathbb{Z}_{+}$(i.e. $(n, m) \in T$ for all $(m, n) \in T)$ containing the diagonal $\left\{(n, n): n \in \mathbb{Z}_{+}\right\}$, and let $Z \subset \mathbb{C}$ be a determining set for $\mathbb{C}[z, \bar{z}]$. Then for any system of complex numbers $\left\{c_{m, n}\right\}_{(m, n) \in T}$, the following conditions are equivalent:

(i) there exists a positive Borel measure $\mu$ on $\mathbb{C}$ such that

$$
c_{m, n}=\int_{\mathbb{C}} z^{m} \bar{z}^{n} \mu(\mathrm{d} z), \quad(m, n) \in T
$$

(ii) there exists a complex moment sequence $\left\{\tilde{c}_{m, n}\right\}_{m, n=0}^{\infty}$ such that $\tilde{c}_{m, n}=c_{m, n}$ for all $(m, n) \in T$,

(iii) there exists $\left\{\tilde{c}_{m, n}\right\}_{m+n \geqslant 0} \subset \mathbb{C}$ such that $\tilde{c}_{m, n}=c_{m, n}$ for all $(m, n) \in T$, and $\sum_{\substack{m+n \geqslant 0 \\ p+q \geqslant 0}} \tilde{c}_{m+q, n+p} \lambda_{m, n} \bar{\lambda}_{p, q} \geqslant 0$ for all finite systems $\left\{\lambda_{m, n}\right\}_{m+n \geqslant 0} \subset$ $\mathbb{C}$,

(iv) $\sum_{(m, n) \in T} p_{m, n} c_{m, n} \geqslant 0$ for every finite system $\left\{p_{m, n}\right\}_{(m, n) \in T} \subset \mathbb{C}$ such that $\sum_{(m, n) \in T} p_{m, n} z^{m} \bar{z}^{n} \geqslant 0$ for all $z \in \mathbb{C}$,

(v) $\sum_{(m, n) \in T} p_{m, n} c_{m, n} \geqslant 0$ for every finite system $\left\{p_{m, n}\right\}_{(m, n) \in T} \subset \mathbb{C}$ for which there exist finitely many rational functions $q_{1}, \ldots, q_{k} \in \mathbb{C}_{\mathfrak{N}_{+}}(z, \bar{z})$ such that $\sum_{(m, n) \in T} p_{m, n} z^{m} \bar{z}^{n}=\sum_{j=1}^{k}\left|q_{j}(z, \bar{z})\right|^{2}$ for all $z \in \mathbb{C}, z \neq 0$,

(vi) $\sum_{(m, n) \in T} p_{m, n} c_{m, n} \geqslant 0$ for every finite system $\left\{p_{m, n}\right\}_{(m, n) \in T} \subset \mathbb{C}$ for which there exist finitely many rational functions $q_{1}, \ldots, q_{k} \in \mathbb{C}_{\mathfrak{N}_{+}}(z, \bar{z})$ such that $\sum_{(m, n) \in T} p_{m, n} z^{m} \bar{z}^{n}=\sum_{j=1}^{k}\left|q_{j}(z, \bar{z})\right|^{2}$ for all $z \in Z, z \neq 0$.

Proof. (i) $\Rightarrow$ (ii) Since $\left\{(n, n): n \in \mathbb{Z}_{+}\right\} \subset T$, we see that complex polynomials in $z$ and $\bar{z}$ are summable with respect to $\mu$. Hence, we can define $\tilde{c}_{m, n}=\int_{\mathbb{C}} z^{m} \bar{z}^{n} \mu(\mathrm{d} z)$ for $m, n \in \mathbb{Z}_{+}$, which is the desired complex moment sequence.

(ii) $\Leftrightarrow$ (iii) This can be deduced from [54, Theorem 1].

Implications (ii) $\Rightarrow(\mathrm{i}),(\mathrm{i}) \Rightarrow(\mathrm{iv})$ and (iv) $\Rightarrow(\mathrm{v})$ are evident.

$(\mathrm{v}) \Rightarrow\left(\right.$ vi) By Lemma 17, $Z \backslash\{0\}$ is a determining set for $\mathbb{C}_{\mathfrak{N}_{+}}(z, \bar{z})$. Since both sides of the equality in (vi) are members of $\mathbb{C}_{\mathfrak{N}_{+}}(z, \bar{z})$, and they coincide on $Z \backslash\{0\}$, we deduce that they are equal on $\mathbb{C}_{*}$.

(vi) $\Rightarrow$ (iii) In view of Lemmata 17 and $18, Y_{Z \backslash\{0\}}$ is a determining set for $\mathscr{P}\left(\mathfrak{X}_{\mathfrak{N}_{+}}\right)$. Since $\mathfrak{N}_{+}$is a semiperfect $*$-semigroup (this can be deduced from 7 [54, Remark 7]), we can apply implication (iv) $\Rightarrow$ (i) of Theorem [15 to $\mathfrak{S}=\mathfrak{N}_{+}, Y=Y_{Z \backslash\{0\}}$ and $\varphi(m, n)=c_{m, n}$. This completes the proof.

Let us point out that there is a difference between the usual meaning of the truncated (complex) moment problem (where a finite system of complex numbers is given) and that appearing in Theorem 19. One of our assumptions requires a system of complex numbers $\left\{c_{m, n}\right\}_{(m, n) \in T}$, which is the given data, to include all the diagonal entries $c_{m, m}, m=0,1,2, \ldots$. This enables us to show that the most direct analogue of the complex version of the Riesz-Haviland theorem for the truncated moment problem in our meaning is true. In the recent paper 19, Example 2.1], Curto and Fialkow have shown that this is not the case for the truncated moment problem in the usual sense. Instead, they have found an analogue of the

\footnotetext{
${ }^{6}$ Notation $m+n \geqslant 0$ has to be understood as $(m, n) \in \mathbb{Z} \times \mathbb{Z}$ and $m+n \geqslant 0$.

7 Indeed, in view of the discussion preceding Lemma 12 we see that our meaning of semiperfectness is wider than that of [54, Remark 7]. The interested reader can verify that for finitely generated unital commutative $*$-semigroups, such as $\mathfrak{N}_{+}$, both these notions are equivalent.
} 
Riesz-Haviland theorem, which requires extending appropriate linear functionals on polynomials of degree limited by $2 n$ to positive linear functionals on polynomials of degree limited by $2 n+2$ (cf. [19, Theorem 2.2]).

The extension procedure required in condition (iii) of Theorem[19 can be realized in two steps: first we have to extend the system $\left\{c_{m, n}\right\}_{(m, n) \in T}$ to a positive definite system over the $*$-semigroup $\mathfrak{N}$, and then to a positive definite system over $\mathfrak{N}_{+}$. The first step can be done in a more explicit way for $*$-ideals $T$ of $\mathfrak{N}$ (i.e. $T=T^{*}$ and $T+\mathfrak{N} \subset T$ ) as in [60; see also [36, 57, 49] for earlier attempts in this direction. However, the $*$-ideal technique is not applicable in the other step when extending positive definite functions from $\mathfrak{N}$ to $\mathfrak{N}_{+}$. This situation requires methods invented in [54]; see Theorem 1 therein.

For further investigation concerning Theorem 19] which exceeds the discussion contained in the present paper, we refer the reader to the subsequent paper [17. In particular, it is shown there that Theorem 19 is no longer true if the assumption that $T$ contains the diagonal $\left\{(n, n): n \in \mathbb{Z}_{+}\right\}$is dropped.

5.1. The lack of symmetry. The phenomenon described below is of a general nature and as such occurs in other instances, such as the truncated multidimensional trigonometric moment problem (cf. Theorem 26) and the truncated two-sided complex moment problem (cf. Theorem 32). Let us discuss it in the case of the truncated complex moment problem, which is related to the $*$-semigroup $\mathfrak{N}_{+}$, and leaving the other cases for the reader.

Consider a not necessarily symmetric set $T$ such that

$$
\left(\mathfrak{N}_{+}\right)_{\mathfrak{h}} \subset T \subset \mathfrak{N}
$$

and look at what happens to the equivalence of the conditions (i)-(vi) of Theorem 19 (the other assumptions of Theorem 19 still being in force). First of all, we see the two natural candidates for replacing $T$ by a symmetric set: $T \cup T^{*}$ and $T \cap T^{*}$, both satisfying (21). As shown below, the set $T \cup T^{*}$ plays an essential role in conditions (i)-(iii) while $T \cap T^{*}$ does so in (iv)-(vi). Because these two sets for very nonsymmetric $T$ 's may differ in the extreme, the aforesaid feature seems to be worthy of taking a closer look at it.

Call a system $\left\{c_{m, n}\right\}_{(m, n) \in T} \subset \mathbb{C}$ symmetrizable if

$$
c_{m, n}=\overline{c_{n, m}}, \quad(m, n) \in T \cap T^{*} .
$$

If $\left\{c_{m, n}\right\}_{(m, n) \in T}$ is symmetrizable, then its symmetrization $\left\{c_{m, n}^{\natural}\right\}_{(m, n) \in T \cup T^{*}}$ :

$$
c_{m, n}^{\natural}= \begin{cases}c_{m, n}, & (m, n) \in T, \\ \overline{c_{n, m}}, & (m, n) \in T^{*},\end{cases}
$$

is well defined. One can verify that if a system $\left\{c_{m, n}\right\}_{(m, n) \in T} \subset \mathbb{C}$ satisfies (i) (respectively: (ii), (iii)) on $T$, then it is symmetrizable and its symmetrization satisfies (i) (respectively: (ii), (iii)) on $T \cup T^{*}$, and vice versa. Theorem 19 implies that, via the symmetrization procedure, for any set $T$ obeying (21) conditions (i)(iii) are equivalent on $T$.

Regarding conditions (iv)-(vi), their prospective equivalence needs to be justified in a different way. Namely, a system $\left\{c_{m, n}\right\}_{(m, n) \in T} \subset \mathbb{C}$ fulfils (iv) (respectively: (v), (vi)) on $T$ if and only if the restricted system $\left\{c_{m, n}\right\}_{(m, n) \in T \cap T^{*}}$ fulfils (iv) (respectively: (v), (vi)) on $T \cap T^{*}$ (hence $c_{m, n}$ 's over $T \backslash T^{*}$ are irrelevant). Indeed, this can be deduced from the fact that a real-valued polynomial $p \in \mathbb{C}[z, \bar{z}]$ belongs 
to $\mathbb{C}_{T}[z, \bar{z}]$ if and only if it belongs to $\mathbb{C}_{T \cap T^{*}}[z, \bar{z}]$ (hint: conjugate the polynomial $p$ and deduce that $p_{m, n}=\overline{p_{n, m}}$, where $p_{m, n}$ are the coefficients of $p$ ). Hence, by Theorem 19] for any set $T$ satisfying (21) conditions (iv)-(vi) are equivalent on $T$. If this happens, then the system $\left\{c_{m, n}\right\}_{(m, n) \in T}$ is symmetrizable.

Since, in fact, conditions (i)-(iii) concern the extension of system $\left\{c_{m, n}\right\}_{(m, n) \in T}$ to $T \cup T^{*}$, while (iv)-(vi) deal with its restriction to $T \cap T^{*}$, it is to be expected that they cannot be altogether equivalent for arbitrary $T$. Indeed, consider any nonsymmetric set $T$ satisfying (21) and take $(k, l) \in T \backslash T^{*}$. Suppose that a system $\left\{c_{m, n}\right\}_{(m, n) \in T} \subset \mathbb{C}$ fulfils conditions (iv)-(vi) on $T$ (e.g. any restriction to $T$ of a complex moment sequence does). Define the new system

$$
\tilde{c}_{m, n}= \begin{cases}c_{m, n}, & (m, n) \in T \backslash\{(k, l)\}, \\ \sqrt{c_{k, k} c_{l, l}+1}, & (m, n)=(k, l) .\end{cases}
$$

Owing to the above discussion the so defined system satisfies conditions (iv)-(vi), but fails to satisfy any of conditions (i)-(iii), because otherwise by the CauchySchwarz inequality we would have $\left|\tilde{c}_{k, l}\right|^{2} \leqslant \tilde{c}_{k, k} \tilde{c}_{l, l}=c_{k, k} c_{l, l}$, a contradiction. This means that Theorem 19 is not true as long as $T$ satisfying (21) is not symmetric. In other words, symmetricity of $T$ is a necessary condition for Theorem 19 to hold.

However, it now becomes clear that for an arbitrary set $T$ satisfying (21) a seemingly more general version of Theorem 19 can be considered. Putting it precisely, for a symmetrizable system $\left\{c_{m, n}\right\}_{(m, n) \in T}$ all of conditions (i)-(vi) remain equivalent if in conditions (iv)-(vi) the system $\left\{c_{m, n}\right\}_{(m, n) \in T}$ is replaced by its symmetrization $\left\{c_{m, n}^{\natural}\right\}_{(m, n) \in T \cup T^{*}}$.

5.2. Sum-square representation. We say that a locally convex topology $\tau$ on the linear space $\mathbb{C}[z, \bar{z}]$ is evaluable if the set

$$
\left\{\lambda \in \mathbb{C} \text { : the evaluation } E_{\lambda}: \mathbb{C}[z, \bar{z}] \ni p \mapsto p(\lambda, \bar{\lambda}) \in \mathbb{C} \text { is } \tau \text {-continuous }\right\}
$$

is dense in $\mathbb{C}$. The class of such topologies is rich. In particular, it contains every locally convex topology on $\mathbb{C}[z, \bar{z}]$ generated by the family $\left\{E_{\lambda}: \lambda \in Z\right\}$, where $Z$ is a dense subset of $\mathbb{C}$. This fact, the linear independence of $\left\{E_{\lambda}: \lambda \in \mathbb{C}\right\}$ and [45. Theorem 3.10] imply that there exist two evaluable topologies such that the only linear functional on $\mathbb{C}[z, \bar{z}]$ continuous with respect to each of them is the zero functional.

One can deduce from Artin's solution of the 17th Hilbert problem (cf. 2 or [13, Theorem 6.1.1]; see also [42, 41] for the case of positive homogeneous polynomials) that for every nonnegative polynomial $p \in \mathbb{C}[z, \bar{z}]$ (i.e. $p(z, \bar{z}) \geqslant 0$ for all $z \in \mathbb{C}$ ), there exist finitely many rational functions $q_{1}, \ldots, q_{n}$ in two complex variables such that $p(z, \bar{z})=\sum_{j=1}^{n}\left|q_{j}(z, \bar{z})\right|^{2}$ for all $z \in \mathbb{C}$ except singularities of the right-hand side of the equality. The question arises whether general rational functions in the above representation of $p$ can be replaced by specific ones from $\mathbb{C}_{\mathfrak{N}_{+}}(z, \bar{z})$. Though we are unable to answer this question in full generality, we can do it in the case in which the nonnegativity of $p$ and its sum-square representation is considered on a closed proper subset $Z$ of $\mathbb{C}$ which is determining for $\mathbb{C}[z, \bar{z}]$. For convenience we denote by $\Sigma^{2}(Z)$ the set of all polynomials $q \in \mathbb{C}[z, \bar{z}]$ for which there exist finitely 
many rational functions $q_{1}, \ldots, q_{n} \in \mathbb{C}_{\mathfrak{N}_{+}}(z, \bar{z})$ such that

$$
q(z, \bar{z})=\sum_{j=1}^{n}\left|q_{j}(z, \bar{z})\right|^{2}, \quad z \in Z \backslash\{0\} .
$$

Perhaps this is the right place to mention that if $q \in \mathbb{C}[z, \bar{z}]$ is of the form (22) with $Z=\mathbb{C}$ and $q_{1}, \ldots, q_{n} \in \mathbb{C}_{\mathbb{Z} \times \mathbb{Z}}(z, \bar{z})$, then by Proposition 16 the rational functions $q_{1}, \ldots, q_{n}$ must belong to $\mathbb{C}_{\mathfrak{N}_{+}}(z, \bar{z})$.

Proposition 20. Let $Z$ be a closed proper subset of $\mathbb{C}$ which is determining for $\mathbb{C}[z, \bar{z}]$ and let $\tau$ be an evaluable locally convex topology on $\mathbb{C}[z, \bar{z}]$. Then there exists a polynomial $p \in \mathbb{C}[z, \bar{z}]$ which is nonnegative on $Z$ and which does not belong to the $\tau$-closure of $\Sigma^{2}(Z)$. In particular, $p$ is not in $\Sigma^{2}(Z)$.

Proof. Denote by $\mathscr{P}^{+}(Z)$ the set of all polynomials $q \in \mathbb{C}[z, \bar{z}]$ which are nonnegative on $Z$ (i.e. $q(z, \bar{z}) \geqslant 0$ for all $z \in Z$ ) and write ${\overline{\Sigma^{2}(Z)}}^{\tau}$ for the $\tau$-closure of $\Sigma^{2}(Z)$. It follows from our assumptions that there exists $\lambda \in \mathbb{C} \backslash Z$ such that the evaluation $E_{\lambda}$ is $\tau$-continuous. By the determining property of $Z \backslash\{0\}$ (cf. Lemma 17), we have $\Sigma^{2}(Z)=\Sigma^{2}(\mathbb{C})$. Hence

$$
E_{\lambda}(q) \geqslant 0, \quad q \in{\overline{\Sigma^{2}(Z)}}^{\tau} .
$$

Since $\lambda \notin Z$, there exists real $\varepsilon>0$ such that $\{z \in \mathbb{C}:|z-\lambda| \leqslant \varepsilon\} \subset \mathbb{C} \backslash Z$. It is then clear that the polynomial $p_{\varepsilon}(z, \bar{z}) \stackrel{\text { def }}{=}|z-\lambda|^{2}-\varepsilon^{2}$ belongs to $\mathscr{P}^{+}(Z)$. Note that $p_{\varepsilon} \notin{\overline{\Sigma^{2}(Z)}}^{\tau}$. Indeed, otherwise (23) implies that $E_{\lambda}\left(p_{\varepsilon}\right) \geqslant 0$, which contradicts $E_{\lambda}\left(p_{\varepsilon}\right)=-\varepsilon^{2}$.

The proof of Proposition 20 remains unchanged if we assume only that $\tau$ is a locally convex topology on $\mathbb{C}[z, \bar{z}]$ for which there exists $\lambda \in \mathbb{C} \backslash Z$ such that the evaluation $E_{\lambda}$ is $\tau$-continuous.

6. Subnormality. Let $S$ be a densely defined linear operator in a complex Hilbert space $\mathcal{H}$ with domain $\mathscr{D}(S)$. We say that $S$ is subnormal if there exist a complex Hilbert space $\mathcal{K}$ and a normal operator $N$ in $\mathcal{K}$ such that $\mathcal{H} \subset \mathcal{K}$ (isometric embedding), $\mathscr{D}(S) \subset \mathscr{D}(N)$ and $S h=N h$ for all $h \in \mathscr{D}(S)$. For fundamentals of the theory of unbounded subnormal operators we refer the reader to [51, 52, 53.

The following characterization of subnormality simplifies substantially that of [52, Theorem 3] (one of the double sums turns out to be redundant). As in [52, it is intrinsic in a sense that no extension is involved. Theorem 21 is also related to part (iv) of [54, Theorem 37] which in the case of $\mathscr{F}=\mathscr{D}$ is equivalent to condition (iii) below.

Theorem 21. Let $S$ be a densely defined linear operator in a complex Hilbert space $\mathcal{H}$ such that $S(\mathscr{D}(S)) \subset \mathscr{D}(S)$. If $Z \subset \mathbb{C}_{*}$ is a determining set for $\mathbb{C}[z, \bar{z}]$, then the following conditions are equivalent:

(i) $S$ is subnormal,

(ii) for every system $\left\{a_{p, q}^{i, j}\right\}_{p, q=0, \ldots, n}^{i, j=1, \ldots, m} \subset \mathbb{C}$, if

$$
\sum_{i, j=1}^{m} \sum_{p, q=0}^{n} a_{p, q}^{i, j} \lambda^{p} \bar{\lambda}^{q} z_{i} \bar{z}_{j} \geqslant 0, \quad \lambda, z_{1}, \ldots, z_{m} \in \mathbb{C}
$$


then

$$
\sum_{i, j=1}^{m} \sum_{p, q=0}^{n} a_{p, q}^{i, j}\left\langle S^{p} f_{i}, S^{q} f_{j}\right\rangle \geqslant 0, \quad f_{1}, \ldots, f_{m} \in \mathscr{D}(S),
$$

(iii) for every system $\left\{a_{p, q}^{i, j}\right\}_{p, q=0, \ldots, n}^{i, j=1, \ldots, m} \subset \mathbb{C}$, if there is a finite matrix $\left[q_{i, l}\right]_{i=1 l=1}^{m, k}$ with entries in $\mathbb{C}_{\mathfrak{N}_{+}}(z, \bar{z})$ such that

$$
\sum_{p, q=0}^{n} a_{p, q}^{i, j} \lambda^{p} \bar{\lambda}^{q}=\sum_{l=1}^{k} q_{i, l}(\lambda, \bar{\lambda}) \overline{q_{j, l}(\lambda, \bar{\lambda})}, \quad \lambda \in Z, i, j=1, \ldots, m,
$$

then (25) holds.

Proof. The proof of implication (i) $\Rightarrow$ (ii) proceeds along the same lines as the proof of the "only if" part of [52, Theorem 3]. The other possibility is to argue as in the proof of Lemma 12(i).

(ii) $\Rightarrow$ (iii) First note that if (26) holds, then, by the determining property of $Z$ (cf. Lemma 17), the equality in (26) is valid for all $\lambda \in \mathbb{C}_{*}$. It is then easily seen that (24) holds, which by (ii) yields (25).

(iii) $\Rightarrow$ (i) Consider the $*$-semigroup $\mathfrak{S}=\mathfrak{N}_{+}$(which is operator semiperfect due to Remark 7 and Proposition 23 in [54]), the linear space $\mathcal{D}=\mathscr{D}(S)$ and the sets $T=\mathbb{Z}_{+} \times \mathbb{Z}_{+}$and $Y=Y_{Z}$ (cf. (18)). Define the mapping $\Phi: T \rightarrow \mathcal{S}(\mathcal{D})$ by

$$
\Phi(m, n)(f, g)=\left\langle S^{m} f, S^{n} g\right\rangle, \quad f, g \in \mathcal{D},(m, n) \in T,
$$

and attach to it the linear mapping $\Lambda_{\Phi, Y}: \mathscr{P}_{T}(Y) \rightarrow \mathcal{S}(\mathcal{D})$ via formula (11). Then a simple calculation based on Lemma 6 shows that (iii) is equivalent to the complete f-positivity of $\Lambda_{\Phi, Y}$. Applying implication (iv) $\Rightarrow$ (i) of Theorem 14] and implication (iii) $\Rightarrow$ (i) of [54, Theorem 37] with $\mathscr{F}=\mathscr{D}$ completes the proof.

7. Unitary dilation of several contractions. In what follows $\varkappa$ stands for a positive integer. Set $\mathbb{Z}_{-}=-\mathbb{Z}_{+}$. Denote by $\mathbb{Z}^{\varkappa}, \mathbb{Z}_{+}^{\varkappa}, \mathbb{Z}_{-}^{\varkappa}$ and $\mathbb{T}^{\varkappa}$ the cartesian product of $\varkappa$ copies of $\mathbb{Z}, \mathbb{Z}_{+}, \mathbb{Z}_{-}$and $\mathbb{T}$, respectively. For simplicity, we write 0 for the zero element of the group $\mathbb{Z}^{\varkappa}$. Observe that $\mathbb{Z}_{+}^{\varkappa} \cup \mathbb{Z}_{-}^{\varkappa}=\mathbb{Z}^{\varkappa}$ only for $\varkappa=1$. Let $\mathscr{P}\left(\mathbb{T}^{\varkappa}\right)$ stand for the linear space of all functions $p: \mathbb{T}^{\varkappa} \rightarrow \mathbb{C}$ of the form

$$
p(z)=\sum_{\alpha \in \mathbb{Z}^{\varkappa}} a_{\alpha} z^{\alpha}, \quad z \in \mathbb{T}^{\varkappa},
$$

where $\left\{a_{\alpha}\right\}_{\alpha \in \mathbb{Z}^{\varkappa}}$ is a finite system of complex numbers, and $z^{\alpha}=z_{1}^{\alpha_{1}} \cdots z_{\varkappa}^{\alpha_{\varkappa}}$ for $z=\left(z_{1}, \ldots, z_{\varkappa}\right) \in \mathbb{T}^{\varkappa}$ and $\alpha=\left(\alpha_{1}, \ldots, \alpha_{\varkappa}\right) \in \mathbb{Z}^{\varkappa}$. The members of $\mathscr{P}\left(\mathbb{T}^{\varkappa}\right)$ are called trigonometric polynomials in $\varkappa$ variables. A trigonometric polynomial $p$ vanishes on $\mathbb{T}^{\varkappa}$ if and only if all its coefficients $a_{\alpha}$ vanish. Given $T \subset \mathbb{Z}^{\varkappa}$, we denote by $\mathscr{P}_{T}\left(\mathbb{T}^{\varkappa}\right)$ the linear space of all trigonometric polynomials $p \in \mathscr{P}\left(\mathbb{T}^{\varkappa}\right)$ of the form (27), where $a_{\alpha}=0$ for all $\alpha \in \mathbb{Z}^{\varkappa} \backslash T$. One can think of members of $\mathscr{P}_{\mathbb{Z}_{+}}\left(\mathbb{T}^{\varkappa}\right)$ as analytic trigonometric polynomials. A nonempty subset $Y$ of $\mathbb{T}^{\varkappa}$ is said to be determining for $\mathscr{P}\left(\mathbb{T}^{\varkappa}\right)$ (respectively: $\mathscr{P}_{\mathbb{Z}_{+}^{\varkappa}}\left(\mathbb{T}^{\varkappa}\right)$ ) if each trigonometric polynomial $p \in \mathscr{P}\left(\mathbb{T}^{\varkappa}\right)$ (respectively: $p \in \mathscr{P}_{\mathbb{Z}_{+}}\left(\mathbb{T}^{\varkappa}\right)$ ) vanishing on $Y$ vanishes on the whole set $\mathbb{T}^{\varkappa}$. Note that any infinite subset of $\mathbb{T}$ is determining for $\mathscr{P}(\mathbb{T})$. 
Lemma 22. A subset $Y$ of $\mathbb{T}^{\varkappa}$ is determining for $\mathscr{P}\left(\mathbb{T}^{\varkappa}\right)$ if and only if it is determining for $\mathscr{P}_{\mathbb{Z}_{+}}\left(\mathbb{T}^{\varkappa}\right)$.

Proof. This is clear, because for every $p \in \mathscr{P}\left(\mathbb{T}^{\varkappa}\right)$ there exists $n \in \mathbb{Z}_{+}$such that the trigonometric polynomial $z_{1}^{n} \ldots z_{\varkappa}^{n} p\left(z_{1}, \ldots, z_{\varkappa}\right)$ is analytic.

Let $\boldsymbol{A}=\left(A_{1}, \ldots, A_{\varkappa}\right)$ be a $\varkappa$-tuple of bounded linear operators on a complex Hilbert space $\mathcal{H}$. Define the family $\left\{\boldsymbol{A}^{[\alpha]}: \alpha \in \mathbb{Z}_{+}^{\varkappa} \cup \mathbb{Z}_{-}^{\varkappa}\right\}$ by

$$
\boldsymbol{A}^{[\alpha]}= \begin{cases}A_{1}^{\alpha_{1}} \cdots A_{\varkappa}^{\alpha_{\varkappa}}, & \alpha \in \mathbb{Z}_{+}^{\varkappa} \\ A_{1}^{*\left|\alpha_{1}\right|} \cdots A_{\varkappa}^{*\left|\alpha_{\varkappa}\right|}, & \alpha \in \mathbb{Z}_{-}^{\varkappa}\end{cases}
$$

For $\alpha \in \mathbb{Z}_{+}^{\varkappa}$ we replace $\boldsymbol{A}^{[\alpha]}$ by the standard multi-index notation $\boldsymbol{A}^{\alpha}$. Following $[62$, page 32], we say that a $\varkappa$-tuple $\boldsymbol{A}$ has a unitary power dilation if there exists a complex Hilbert space $\mathcal{K} \supset \mathcal{H}$ (isometric embedding) and a $\varkappa$-tuple $\boldsymbol{U}=\left(U_{1}, \ldots, U_{\varkappa}\right)$ of commuting unitary operators on $\mathcal{K}$ such that

$$
\boldsymbol{A}^{\alpha}=\left.P \boldsymbol{U}^{\alpha}\right|_{\mathcal{H}}, \quad \alpha \in \mathbb{Z}_{+}^{\varkappa},
$$

where $P$ stands for the orthogonal projection of $\mathcal{K}$ onto $\mathcal{H}$. Such $\boldsymbol{U}$ is called a unitary power dilation of $\boldsymbol{A}$. The proof of the following fact is left to the reader.

Lemma 23. If $\boldsymbol{U}$ is a unitary power dilation of $\boldsymbol{A}$, then the operators $A_{1}, \ldots, A_{\varkappa}$ commute if and only if $\boldsymbol{A}^{[\alpha]}=\left.P \boldsymbol{U}^{\alpha}\right|_{\mathcal{H}}$ for all $\alpha \in \mathbb{Z}_{+}^{\varkappa} \cup \mathbb{Z}_{-}^{\varkappa}$.

We are now in a position to formulate necessary and sufficient conditions for a $\varkappa$ tuple of bounded operators to have a unitary power dilation. Theorem 24 below is related to characterizations of families of operators having unitary power dilations given in [55, Corollary 6] (see also [33, Lemma 1] for another formulation which does not appeal to the boundedness of $\boldsymbol{A}$; in fact, one can easily write a version of Theorem 24 for operators which are not a priori assumed to be bounded). Different approaches to the problem of the existence of unitary power dilation have recently appeared in 63. and 1]; however, the solutions proposed therein are not written in terms of the operators in question.

Theorem 24. If $\boldsymbol{A}=\left(A_{1}, \ldots, A_{\varkappa}\right)$ is a $\varkappa$-tuple of bounded linear operators on a complex Hilbert space $\mathcal{H}$ and $Y$ is a determining set for $\mathscr{P}_{\mathbb{Z}_{+}^{\varkappa}}\left(\mathbb{T}^{\varkappa}\right)$, then the following conditions are equivalent:

(i) $\boldsymbol{A}$ has a unitary power dilation and the operators $A_{1}, \ldots, A_{\varkappa}$ commute,

(ii) for every finite system $\left\{a_{\alpha}^{i, j}: i, j=1, \ldots, m, \alpha \in \mathbb{Z}_{+}^{\varkappa} \cup \mathbb{Z}_{-}^{\varkappa}\right\} \subset \mathbb{C}$, if

$$
\sum_{i, j=1}^{m} \sum_{\alpha \in \mathbb{Z}_{+}^{\varkappa} \cup \mathbb{Z}_{-}^{\varkappa}} a_{\alpha}^{i, j} \lambda^{\alpha} z_{i} \bar{z}_{j} \geqslant 0, \quad \lambda \in \mathbb{T}^{\varkappa}, z_{1}, \ldots, z_{m} \in \mathbb{C},
$$

then

$$
\sum_{i, j=1}^{m} \sum_{\alpha \in \mathbb{Z}_{+}^{\varkappa} \cup \mathbb{Z}_{-}^{\varkappa}} a_{\alpha}^{i, j}\left\langle\boldsymbol{A}^{[\alpha]} f_{i}, f_{j}\right\rangle \geqslant 0, \quad f_{1}, \ldots, f_{m} \in \mathcal{H},
$$


(iii) for every finite system $\left\{a_{\alpha}^{i, j}: i, j=1, \ldots, m, \alpha \in \mathbb{Z}_{+}^{\varkappa} \cup \mathbb{Z}_{-}^{\varkappa}\right\} \subset \mathbb{C}$, if there is a finite matrix $\left[q_{i, l}\right]_{i=1 l=1}^{m}$ with entries in $\mathscr{P}_{\mathbb{Z}_{+}^{\varkappa}}\left(\mathbb{T}^{\varkappa}\right)$ such that

$$
\sum_{\alpha \in \mathbb{Z}_{+}^{\varkappa} \cup \mathbb{Z}_{-}^{\varkappa}} a_{\alpha}^{i, j} \lambda^{\alpha}=\sum_{l=1}^{k} q_{i, l}(\lambda) \overline{q_{j, l}(\lambda)}, \quad \lambda \in Y, i, j=1, \ldots, m,
$$

then (29) holds.

Proof. Without loss of generality we can assume that $Y$ is a determining set for $\mathscr{P}\left(\mathbb{T}^{\varkappa}\right)$ (cf. Lemma 22). In what follows, we regard $\mathbb{Z}^{\varkappa}$ as the $*$-semigroup equipped with coordinatewise defined addition as semigroup operation and involution $\alpha^{*}=$ $-\alpha, \alpha \in \mathbb{Z}^{\varkappa}$. In turn, $\mathbb{T}^{\varkappa}$ is regarded as the multiplicative $*$-semigroup with coordinatewise defined multiplication as semigroup operation and involution

$$
\left(z_{1}, \ldots, z_{\varkappa}\right)^{*}=\left(\bar{z}_{1}, \ldots, \bar{z}_{\varkappa}\right), \quad\left(z_{1}, \ldots, z_{\varkappa}\right) \in \mathbb{T}^{\varkappa}
$$

It is easily checked that the dual $*$-semigroup $\mathfrak{X}_{\mathbb{Z}^{\varkappa}}$ of $\mathbb{Z}^{\varkappa}$ can be identified algebraically with the $*$-semigroup $\mathbb{T}^{\varkappa}$ via the mapping

$$
\mathfrak{X}_{\mathbb{Z}^{\varkappa}} \ni \chi \mapsto\left(\chi\left(e_{1}\right), \ldots, \chi\left(e_{\varkappa}\right)\right) \in \mathbb{T}^{\varkappa},
$$

where $e_{j}=\left(\delta_{j, 1}, \ldots, \delta_{j, \varkappa}\right) \in \mathbb{Z}^{\varkappa}\left(\delta_{i, j}\right.$ is the Kronecker delta function). Under this identification, $\widehat{\alpha}$ is given by

$$
\widehat{\alpha}(z)=z^{\alpha}, \quad z=\left(z_{1}, \ldots, z_{\varkappa}\right) \in \mathbb{T}^{\varkappa}, \alpha \in \mathbb{Z}^{\varkappa},
$$

which means that the notation $\mathscr{P}\left(\mathbb{T}^{\varkappa}\right)$ introduced at the beginning of this section is consistent with that for $*$-semigroups in Section 1 and that $Y$ is a determining subset of $\mathfrak{X}_{\mathbb{Z}^{\varkappa}}$. It is well known that the $*$-semigroup $\mathbb{Z}^{\varkappa}$ is operator semiperfect (e.g. see [37] and footnote [7). Put $T=\mathbb{Z}_{+}^{\varkappa} \cup \mathbb{Z}_{-}^{\varkappa}$ and define $\Phi: T \rightarrow \mathcal{S}(\mathcal{H})$ via

$$
\Phi(\alpha)(f, g)=\left\langle\boldsymbol{A}^{[\alpha]} f, g\right\rangle, \quad f, g \in \mathcal{H}, \alpha \in T .
$$

In view of Lemma 23 and [55, Theorem 3], the current condition (i) is equivalent to condition (i) of Theorem 14 with $\mathfrak{S}=\mathbb{Z}^{\varkappa}$ and $\mathcal{D}=\mathcal{H}$. In turn, the current condition (ii) is a counterpart of condition (iii) of Theorem 14. Finally, by Lemma 6. the current condition (iii) is a counterpart of condition (iv) of Theorem 14, because if (30) holds for some matrix $\left[q_{i, l}\right]_{i=1 l=1}^{m}$ with entries in $\mathscr{P}\left(\mathbb{T}^{\varkappa}\right)$, then there exists $n \in \mathbb{Z}_{+}$such that all the trigonometric polynomials

$$
\tilde{q}_{i, l}\left(z_{1}, \ldots, z_{\varkappa}\right) \stackrel{\text { def }}{=} z_{1}^{n} \ldots z_{\varkappa}^{n} q_{i, l}\left(z_{1}, \ldots, z_{\varkappa}\right), \quad z_{1}, \ldots, z_{\varkappa} \in \mathbb{T},
$$

are analytic and (30) is valid with $\left[\tilde{q}_{i, l}\right]_{i=1}^{m} \underset{l=1}{k}$ in place of $\left[q_{i, l}\right]_{i=1}^{m} \underset{l=1}{k}$. Hence, applying Theorem 14 completes the proof.

Remark 25. The implication (iii) $\Longrightarrow$ (ii) of Theorem 24 can also be deduced from [20. Corollary 5.2]. Indeed, if (28) is valid, then for every real $\varepsilon>0$, the squarematrix-valued trigonometric polynomial $Q^{(\varepsilon)}(\lambda) \stackrel{\text { def }}{=} \sum_{\alpha \in \mathbb{Z}_{+} \cup \mathbb{Z}_{-}^{\varkappa}} Q_{\alpha} \lambda^{\alpha}+\varepsilon I_{m}$, where $Q_{\alpha}=\left[a_{\alpha}^{i, j}\right]_{i, j=1}^{m}$ and $I_{m}=\left[\delta_{i, j}\right]_{i, j=1}^{m}$, is strictly positive on $\mathbb{T}^{\varkappa}$. By [20, Corollary $5.2]$, the polynomial $Q^{(\varepsilon)}$ has a factorization by an analytic (in general nonsquare) matrix-valued trigonometric polynomial. This implies that the polynomial $Q^{(\varepsilon)}$ 
takes the form which is required in (30) (use the trick 8 from the proof of Lemma 221). Hence, by (iii), we have

$$
\sum_{i, j=1}^{m} \sum_{\alpha \in \mathbb{Z}_{+} \cup \mathbb{Z}_{-}^{\varkappa}} a_{\alpha}^{i, j}\left\langle\boldsymbol{A}^{[\alpha]} f_{i}, f_{j}\right\rangle+\varepsilon \sum_{i=1}^{m}\left\|f_{i}\right\|^{2} \geqslant 0, \quad f_{1}, \ldots, f_{m} \in \mathcal{H}, \varepsilon>0 .
$$

Passing with $\varepsilon$ to 0 completes the proof.

8. The truncated multidimensional trigonometric moment problem. The problem in the title goes back to Krein's theorem [35] on extending positive definite functions from an interval to $\mathbb{R}$. A several dimensional version of this is not true according to examples of Calderón and Pepinsky [16] (the additive group $\mathbb{Z}^{\varkappa}$ ) and Rudin [44] (the additive groups $\mathbb{Z}^{\varkappa}$ and $\mathbb{R}^{\varkappa}$ ). In this section we concentrate on the discrete case. Our result, which is related to [55, Corollary 4], deals with the truncated multidimensional trigonometric moment problem on symmetric subsets of $\mathbb{Z}^{\varkappa}$.

It was proved in [16], and independently in [44, that a finite nonempty subset $\Lambda$ of $\mathbb{Z}^{\varkappa}$ has the extension property (i.e. each complex function on $\Lambda-\Lambda$ which is positive definite on $\Lambda$ extends to a positive definite complex function on $\mathbb{Z}^{\varkappa}$ ) if and only if each nonnegative trigonometric polynomial in $\mathscr{P}_{\Lambda-\Lambda}\left(\mathbb{T}^{\varkappa}\right)$ is equal to a finite sum of squares of moduli of trigonometric polynomials in $\mathscr{P}_{\Lambda}\left(\mathbb{T}^{\varkappa}\right)$. Recently, Gabardo found new conditions under which $\Lambda$ has or fails to have the extension property (cf. 25]; see also 26] for $\Lambda$-determinacy and 23, 24] for related questions). Clearly, if $\Lambda$ has the extension property, then $\mathscr{P}_{\Lambda-\Lambda}^{+}\left(\mathbb{T}^{\varkappa}\right)=\Sigma_{\Lambda-\Lambda}^{2}\left(\mathbb{T}^{\varkappa}\right)$ (see Section 9 for notation). Note that each difference set $T \subset \mathbb{Z}^{\varkappa}$, i.e. a set of the form $\Lambda-\Lambda$ with some nonempty $\Lambda \subset \mathbb{R}^{\varkappa}$, has the property $0 \in T=-T$, which is required in Theorem 26 below. However, not every set $T \subset \mathbb{Z}^{\varkappa}$ satisfying $0 \in T=-T$ is a difference set, which can be seen even for $\varkappa=1$. Namely, one can show that for all integers $k, n$ such that $1 \leqslant k<n-k<n$ (necessarily $n \geqslant 3$ and $k<n / 2$ ), any subset $T$ of $\mathbb{Z}$ fulfilling the conditions

(i) $0 \in T=-T$,

(ii) $T \subset\{j \in \mathbb{Z}:|j| \leqslant n\}$,

(iii) $n-k, n \in T$,

(iv) $k \notin T$ and $j \notin T$ for every integer $j$ such that $n-k<j<n$,

is not a difference set (hint: replace $\Lambda$ by $\Lambda-\min \Lambda$ ). The cardinality of such sets $T$ may (and does) vary between 5 and $2(n-k)+1$. There is a simple way of producing multidimensional variants of nondifference sets from one dimensional ones. Indeed, if $T_{1}$ is a nondifference subset of $\mathbb{Z}$ and $T^{\prime}$ is any subset of $\mathbb{Z}^{\varkappa}$, then $T_{1} \times T^{\prime}$ is a nondifference subset of $\mathbb{Z}^{\varkappa+1}$ (hint: $P(\Lambda-\Lambda)=P(\Lambda)-P(\Lambda)$, where $P(n, \alpha)=n$ for $n \in \mathbb{Z}$ and $\left.\alpha \in \mathbb{Z}^{\varkappa}\right)$. Of course, there are nondifference sets which cannot be obtained this way, e.g. $\varkappa=2$ and $T=\{(0,0),(1,0),(-1,0),(1,1),(-1,-1)\}$. Summing up, our solutions of the truncated trigonometric moment problem given in Theorem 26 below allow for much more general truncations, even in the case of finite data $T$. Surprisingly, the infinite set $\mathbb{Z}_{+}^{\varkappa} \cup \mathbb{Z}_{-}^{\varkappa}$, playing a pivotal role in Section 7. is a difference set. Indeed, if $\mathbb{Z}_{+} \ni n \mapsto \alpha_{n} \in \mathbb{Z}_{+}^{\varkappa}$ is any surjection with $\alpha_{0}=0$,

${ }^{8}$ It is required because Dritschel's factorization $F(\lambda)^{*} F(\lambda)$, as in 20], differs from the factorization $P(\lambda) P(\lambda)^{*}$ involved in (30) by the location of the asterisk. 
then $\mathbb{Z}_{+}^{\varkappa} \cup \mathbb{Z}_{-}^{\varkappa}=\Lambda-\Lambda$ with

$$
\Lambda=\left\{\alpha_{0}+\ldots+\alpha_{n}: n \in \mathbb{Z}_{+}\right\} .
$$

Another (more explicit) choice of $\Lambda$ for $\varkappa=2$ is illustrated in Figure 2.

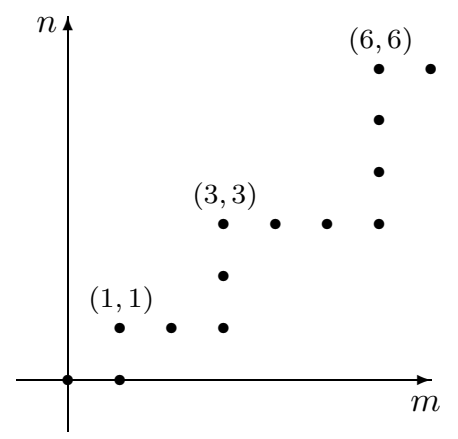

Figure 2. An example of $\Lambda$ such that $\mathbb{Z}_{+}^{2} \cup \mathbb{Z}_{-}^{2}=\Lambda-\Lambda$.

We now go back to solving the truncated trigonometric moment problem.

Theorem 26. Assume that $T$ is a subset of $\mathbb{Z}^{\varkappa}$ such that $0 \in T=-T$, and $Y$ is a determining set for $\mathscr{P}_{\mathbb{Z}_{+}^{\varkappa}}\left(\mathbb{T}^{\varkappa}\right)$. If $\left\{c_{\alpha}\right\}_{\alpha \in T}$ is a system of complex numbers, then the following conditions are equivalent:

(i) there exists a finite positive Borel measure $\mu$ on $\mathbb{T}^{\varkappa}$ such that

$$
c_{\alpha}=\int_{\mathbb{T}^{\varkappa}} z^{\alpha} \mathrm{d} \mu(z), \quad \alpha \in T
$$

(ii) $\sum_{\alpha \in T} a_{\alpha} c_{\alpha} \geqslant 0$ for every finite system $\left\{a_{\alpha}\right\}_{\alpha \in T}$ of complex numbers such that $\sum_{\alpha \in T} a_{\alpha} z^{\alpha} \geqslant 0$ for all $z \in \mathbb{T}^{\varkappa}$,

(iii) $\sum_{\alpha \in T} a_{\alpha} c_{\alpha} \geqslant 0$ for every finite system $\left\{a_{\alpha}\right\}_{\alpha \in T}$ of complex numbers for which there exist finitely many analytic trigonometric polynomials $q_{1}, \ldots, q_{k}$ $\in \mathscr{P}_{\mathbb{Z}_{+}^{\varkappa}}\left(\mathbb{T}^{\varkappa}\right)$ such that $\sum_{\alpha \in T} a_{\alpha} z^{\alpha}=\sum_{j=1}^{k}\left|q_{j}(z)\right|^{2}$ for all $z \in Y$.

Proof. We can argue essentially as in the proof of Theorem 24 using Theorem [15] instead of Theorem 14

It is worth mentioning that Proposition 20 can be easily adapted to the present context. We say that a locally convex topology $\tau$ on the linear space $\mathscr{P}_{\mathbb{Z}_{+}^{\varkappa} \cup \mathbb{Z}_{-}^{\varkappa}}\left(\mathbb{T}^{\varkappa}\right)$ (cf. Section 7) is evaluable if the set of all points $\lambda \in \mathbb{T}^{\varkappa}$ for which the evaluation

$$
\mathscr{P}_{\mathbb{Z}_{+} \cup \mathbb{Z}_{-}^{\varkappa}}\left(\mathbb{T}^{\varkappa}\right) \ni p \mapsto p(\lambda) \in \mathbb{C}
$$

is $\tau$-continuous is dense in $\mathbb{T}^{\varkappa}$. Given $Y \subset \mathbb{T}^{\varkappa}$, we denote by $\Sigma^{2}(Y)$ the set of all trigonometric polynomials $q \in \mathscr{P}_{\mathbb{Z}_{+} \cup \mathbb{Z}_{-}^{\varkappa}}\left(\mathbb{T}^{\varkappa}\right)$ for which there exist finitely many analytic trigonometric polynomials $q_{1}, \ldots, q_{n} \in \mathscr{P}_{\mathbb{Z}_{+}^{\varkappa}}\left(\mathbb{T}^{\varkappa}\right)$ such that

$$
q(z)=\sum_{j=1}^{n}\left|q_{j}(z)\right|^{2}, \quad z \in Y
$$

\footnotetext{
${ }^{9}$ This is the explicit form of condition (13) under the circumstances of the $*$-semigroup $\mathbb{Z}^{\varkappa}$.
} 
Arguing as in the proof of Proposition 20 with $p_{\varepsilon} \in \mathscr{P}_{\mathbb{Z}_{+}^{\varkappa} \cup \mathbb{Z}_{-}^{\varkappa}}\left(\mathbb{T}^{\varkappa}\right)$ given by

$p_{\varepsilon}(z)=\sum_{j=1}^{\varkappa}\left|z_{j}-\lambda_{j}\right|^{2}-\varepsilon^{2}=2 \varkappa-\varepsilon^{2}-\sum_{j=1}^{\varkappa} \bar{\lambda}_{j} z_{j}-\sum_{j=1}^{\varkappa} \lambda_{j} z_{j}^{-1}, \quad z=\left(z_{1}, \ldots, z_{\varkappa}\right) \in \mathbb{T}^{\varkappa}$, where $\left(\lambda_{1}, \ldots, \lambda_{\varkappa}\right) \in \mathbb{T}^{\varkappa} \backslash Y$, we are led to the ensuing result.

Proposition 27. Let $Y$ be a closed proper subset of $\mathbb{T}^{\varkappa}$ which is determining for

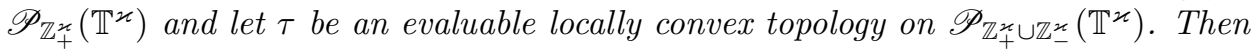
there exists a trigonometric polynomial $p \in \mathscr{P}_{\mathbb{Z}_{+}^{\varkappa} \cup \mathbb{Z}_{-}^{\varkappa}}\left(\mathbb{T}^{\varkappa}\right)$ which is nonnegative on $Y$ and which does not belong to the $\tau$-closure of $\Sigma^{2}(Y)$. In particular, $p$ is not in $\Sigma^{2}(Y)$.

Note that if a trigonometric polynomial $p \in \mathscr{P}(\mathbb{T})=\mathscr{P}_{\mathbb{Z}_{+} \cup \mathbb{Z}_{-}}(\mathbb{T})$ is nonnegative on $\mathbb{T}$, then by the Féjer-Riesz theorem there exists an analytic trigonometric polynomial $q \in \mathscr{P}_{\mathbb{Z}_{+}}(\mathbb{T})$ such that $p(z)=|q(z)|^{2}$ for all $z \in \mathbb{T}$.

9. Approximation. In this section we intend to apply our approach to approximating nonnegative polynomials by sums of squares of moduli of rational functions. The method presented here could be a fertile source of many other approximation results of this kind.

Let $T$ be a subset of $\mathbb{Z}_{+} \times \mathbb{Z}_{+}$. Denote by $\Sigma_{T}^{2}(\mathbb{C})$ the set of all polynomials $q \in$ $\mathbb{C}_{T}[z, \bar{z}]$ for which there exist finitely many rational functions $q_{1}, \ldots, q_{k} \in \mathbb{C}_{\mathfrak{N}_{+}}(z, \bar{z})$ such that $q(z, \bar{z})=\sum_{j=1}^{k}\left|q_{j}(z, \bar{z})\right|^{2}$ for all $z \in \mathbb{C}_{*}$. Let $\mathscr{P}_{T}^{+}(\mathbb{C})$ stand for the set of all polynomials $q \in \mathbb{C}_{T}[z, \bar{z}]$ such that $q(z, \bar{z}) \geqslant 0$ for all $z \in \mathbb{C}$. We shall regard $\Sigma_{T}^{2}(\mathbb{C}), \mathscr{P}_{T}^{+}(\mathbb{C})$ and $\mathbb{C}_{T}[z, \bar{z}]$ as sets of complex functions on $\mathbb{C}$. Given $p \in \mathbb{C}[z, \bar{z}]$ of the form $p(z, \bar{z})=\sum_{m, n \geqslant 0} a_{m, n} z^{m} \bar{z}^{n}$, we set $\|p\|_{\text {co }}=\max \left\{\left|a_{m, n}\right|: m, n \geqslant 0\right\}$. The function $\|\cdot\|_{\text {co }}$ is a norm on $\mathbb{C}[z, \bar{z}]$. Recall that the finest locally convex topology on a linear space $V$ is given by the family of all seminorms on $V$.

Proposition 28. Let $T$ be a subset of $\mathbb{Z}_{+} \times \mathbb{Z}_{+}$such that $(n, m) \in T$ for all $(m, n) \in T$, and $(n, n) \in T$ for all $n \in \mathbb{Z}_{+}$. Then the set $\Sigma_{T}^{2}(\mathbb{C})$ is dense in $\mathscr{P}_{T}^{+}(\mathbb{C})$ with respect to the finest locally convex topology $\tau$ on $\mathbb{C}_{T}[z, \bar{z}]$. In particular, this is the case for the topology of uniform convergence on compact subsets of $\mathbb{C}$ and the topology given by the norm $\|\cdot\|_{\mathrm{co}}$.

Proof. It only suffices to discuss the case of the finest locally convex topology. We regard $\mathbb{C}[z, \bar{z}]$ as a $*$-algebra of complex functions on $\mathbb{C}$ with involution $q^{*}(z, \bar{z})=$ $\overline{q(z, \bar{z})}$ for $z \in \mathbb{C}$. Since the set $T$ is assumed to have a symmetry property, we see that $\mathbb{C}_{T}[z, \bar{z}]$ is a vector subspace of $\mathbb{C}[z, \bar{z}]$ such that $q^{*} \in \mathbb{C}_{T}[z, \bar{z}]$ for every $q \in \mathbb{C}_{T}[z, \bar{z}]$. It is then clear that

$$
\Sigma_{T}^{2}(\mathbb{C}) \subset \mathscr{P}_{T}^{+}(\mathbb{C}) \subset \mathbb{C}_{T}[z, \bar{z}]_{\mathfrak{h}} \stackrel{\text { def }}{=}\left\{q \in \mathbb{C}_{T}[z, \bar{z}]: q=q^{*}\right\} .
$$

Suppose that, contrary to our claim, there exists $q_{0} \in \mathscr{P}_{T}^{+}(\mathbb{C})$ which does not belong to the $\tau$-closure of $\Sigma_{T}^{2}(\mathbb{C})$. By the separation theorem (cf. [45, Theorem 3.4(b)]), there exist a real-linear functional $\tilde{\Lambda}: \mathbb{C}_{T}[z, \bar{z}] \rightarrow \mathbb{R}$ and $\gamma \in \mathbb{R}$ such that

$$
\tilde{\Lambda}\left(q_{0}\right)<\gamma \leqslant \tilde{\Lambda}(q), \quad q \in \Sigma_{T}^{2}(\mathbb{C}) .
$$

Since $\Sigma_{T}^{2}(\mathbb{C})$ has the property that $t q \in \Sigma_{T}^{2}(\mathbb{C})$ for all $q \in \Sigma_{T}^{2}(\mathbb{C})$ and $t \in[0, \infty)$, we deduce from (31) that

$$
\tilde{\Lambda}\left(q_{0}\right)<0 \leqslant \tilde{\Lambda}(q), \quad q \in \Sigma_{T}^{2}(\mathbb{C}) .
$$


Define $\Lambda: \mathbb{C}_{T}[z, \bar{z}] \rightarrow \mathbb{C}$ by

$$
\Lambda(q)=\tilde{\Lambda}(\mathfrak{R e} q)+\mathrm{i} \tilde{\Lambda}(\mathfrak{I m} q), \quad q \in \mathbb{C}_{T}[z, \bar{z}],
$$

with $\mathfrak{R e} q \stackrel{\text { def }}{=}\left(q+q^{*}\right) / 2$ and $\mathfrak{I m} q \stackrel{\text { def }}{=}\left(q-q^{*}\right) /(2 \mathrm{i})$. It is easily seen that $\Lambda$ is a complex-linear functional such that $\Lambda(q)=\tilde{\Lambda}(q)$ for all $q \in \mathbb{C}_{T}[z, \bar{z}]_{\mathfrak{h}}$. Hence, in view of the inclusion $\left\{q_{0}\right\} \cup \Sigma_{T}^{2}(\mathbb{C}) \subset \mathbb{C}_{T}[z, \bar{z}]_{\mathfrak{h}}$, we have

$$
\Lambda\left(q_{0}\right)<0 \leqslant \Lambda(q), \quad q \in \Sigma_{T}^{2}(\mathbb{C}) .
$$

Since $\Lambda$ is of the form

$$
\Lambda(q)=\sum_{(m, n) \in T} p_{m, n} c_{m, n} \quad \text { for } \quad q \in \mathbb{C}_{T}[z, \bar{z}]: q(z, \bar{z})=\sum_{(m, n) \in T} p_{m, n} z^{m} \bar{z}^{n},
$$

where $\left\{c_{m, n}\right\}_{(m, n) \in T}$ is a system of complex numbers uniquely determined by $\Lambda$, we deduce from the weak inequality in (32) and implication (v) $\Rightarrow(\mathrm{iv})$ of Theorem 19 that $\Lambda\left(q_{0}\right) \geqslant 0$, which contradicts the strict inequality in (32).

Now consider the case of $T=\mathfrak{N}=\mathbb{Z}_{+} \times \mathbb{Z}_{+}$. Observe that $\widetilde{\Sigma}_{\mathfrak{N}}^{2}(\mathbb{C})$, the convex cone of all polynomials $q \in \mathbb{C}[z, \bar{z}]$ for which there exist finitely many polynomials $p_{1}, \ldots, p_{n} \in \mathbb{C}[z, \bar{z}]$ such that $q(z, \bar{z})=\sum_{j=1}^{n}\left|p_{j}(z, \bar{z})\right|^{2}$ for all $z \in \mathbb{C}$, is closed with respect to the finest locally convex topology of $\mathbb{C}[z, \bar{z}]$ (see the proof of $[5$, Theorem 6.3.5]). Evidently, the convex cone $\mathscr{P}_{\mathfrak{N}}^{+}(\mathbb{C})$ is closed with respect to the same topology. It is clear that

$$
\widetilde{\Sigma}_{\mathfrak{N}}^{2}(\mathbb{C}) \subset \Sigma_{\mathfrak{N}}^{2}(\mathbb{C}) \subset \mathscr{P}_{\mathfrak{N}}^{+}(\mathbb{C}) .
$$

Owing to the proof of [5, Theorem 6.3.5], $\widetilde{\Sigma}_{\mathfrak{N}}^{2}(\mathbb{C})$ is a proper subset of $\mathscr{P}_{\mathfrak{N}}^{+}(\mathbb{C})$. Hence, by Proposition 28, the first inclusion in (33) is proper as well. The open question as to whether the second inclusion in (33) is proper has already been posed in the paragraph preceding Proposition 20.

Corollary 29. Let

$$
q(z, \bar{z})=\sum_{m, n \geqslant 0} a_{m, n} z^{m} \bar{z}^{n}, \quad z \in \mathbb{C},
$$

be a nonnegative polynomial with coefficients in $\mathbb{C}$. Then for every $\varepsilon>0$, there exists a nonnegative polynomial $q_{\varepsilon} \in \Sigma_{\mathfrak{N}}^{2}(\mathbb{C})$ such that

$$
\begin{aligned}
& q_{\varepsilon}(z, \bar{z})=\sum_{m, n \geqslant 0} a_{m, n}^{(\varepsilon)} z^{m} \bar{z}^{n}, \quad z \in \mathbb{C} \quad\left(a_{m, n}^{(\varepsilon)} \in \mathbb{C}\right), \\
& \left|a_{m, n}-a_{m, n}^{(\varepsilon)}\right| \leqslant \varepsilon\left|a_{m, n}\right|, \quad m, n \in \mathbb{Z}_{+}, m \neq n, \\
& \left|a_{m, n}-a_{m, n}^{(\varepsilon)}\right| \leqslant \varepsilon, \quad m, n \in \mathbb{Z}_{+} .
\end{aligned}
$$

Proof. It is enough to consider the case of $q \neq 0$. Since the polynomial $q$ is nonnegative, we deduce that the set

$$
T=\left\{(m, n) \in \mathbb{Z}_{+} \times \mathbb{Z}_{+}: a_{m, n} \neq 0\right\} \cup\left\{(n, n): n \in \mathbb{Z}_{+}\right\}
$$

satisfies the assumptions of Proposition 28, and $q \in \mathscr{P}_{T}^{+}(\mathbb{C})$. By this proposition, there exists $q_{\varepsilon} \in \Sigma_{T}^{2}(\mathbb{C})$ such that $\left\|q-q_{\varepsilon}\right\|_{\text {co }} \leqslant \tilde{\varepsilon}$, where

$$
\tilde{\varepsilon}=\varepsilon \cdot \min \left\{1, \min \left\{\left|a_{m, n}\right|: m, n \geqslant 0, a_{m, n} \neq 0\right\}\right\}>0 .
$$

This completes the proof. 
We now discuss the case of trigonometric polynomials (see Section 7 for notation). Let $T$ be a subset of $\mathbb{Z}^{\varkappa}$ and let $\Sigma_{T}^{2}\left(\mathbb{T}^{\varkappa}\right)$ stand for the set of all trigonometric polynomials $q \in \mathscr{P}_{T}\left(\mathbb{T}^{\varkappa}\right)$ for which there exist finitely many analytic trigonometric polynomials $q_{1}, \ldots, q_{k} \in \mathscr{P}_{\mathbb{Z}_{+}^{\varkappa}}\left(\mathbb{T}^{\varkappa}\right)$ such that $q(z)=\sum_{j=1}^{k}\left|q_{j}(z)\right|^{2}$ for all $z \in \mathbb{T}^{\varkappa}$. Denote by $\mathscr{P}_{T}^{+}\left(\mathbb{T}^{\varkappa}\right)$ the set of all trigonometric polynomials $q \in \mathscr{P}_{T}\left(\mathbb{T}^{\varkappa}\right)$ such that $q(z) \geqslant 0$ for all $z \in \mathbb{T}^{\varkappa}$. Given $p \in \mathscr{P}\left(\mathbb{T}^{\varkappa}\right)$ of the form (27), we set $\|p\|_{\text {co }}^{\prime}=$ $\max \left\{\left|a_{\alpha}\right|: \alpha \in \mathbb{Z}^{\varkappa}\right\}$. It is clear that $\|\cdot\|_{\text {co }}^{\prime}$ is a norm on $\mathscr{P}\left(\mathbb{T}^{\varkappa}\right)$.

Arguing as in the proof of Proposition 28 (using Theorem 26]instead of Theorem 19), we get another approximation result.

Proposition 30. Let $T$ be a subset of $\mathbb{Z}^{\varkappa}$ such that $0 \in T=-T$. Then the set $\Sigma_{T}^{2}\left(\mathbb{T}^{\varkappa}\right)$ is dense in $\mathscr{P}_{T}^{+}\left(\mathbb{T}^{\varkappa}\right)$ with respect to the finest locally convex topology on $\mathscr{P}_{T}\left(\mathbb{T}^{\varkappa}\right)$. In particular, this is the case for the topology of uniform convergence on $\mathbb{T}^{\varkappa}$ and the topology given by the norm $\|\cdot\|_{\mathrm{co}}^{\prime}$.

Corollary 31. Let

$$
q(z)=\sum_{\alpha \in \mathbb{Z}^{\varkappa}} a_{\alpha} z^{\alpha}, \quad z \in \mathbb{T}^{\varkappa}
$$

be a nonnegative trigonometric polynomial with coefficients in $\mathbb{C}$. Then for every $\varepsilon>0$, there exists a nonnegative trigonometric polynomial $q_{\varepsilon} \in \Sigma_{\mathbb{Z}^{\varkappa}}^{2}\left(\mathbb{T}^{\varkappa}\right)$ such that

$$
\begin{aligned}
& q_{\varepsilon}(z)=\sum_{\alpha \in \mathbb{Z}^{\varkappa}} a_{\alpha}^{(\varepsilon)} z^{\alpha}, \quad z \in \mathbb{T}^{\varkappa} \quad\left(a_{\alpha}^{(\varepsilon)} \in \mathbb{C}\right), \\
& \left|a_{\alpha}-a_{\alpha}^{(\varepsilon)}\right| \leqslant \varepsilon\left|a_{\alpha}\right|, \quad \alpha \in \mathbb{Z}^{\varkappa} \backslash\{(0, \ldots, 0)\},
\end{aligned}
$$

and

$$
\left|a_{\alpha}-a_{\alpha}^{(\varepsilon)}\right| \leqslant \varepsilon, \quad \alpha \in \mathbb{Z}^{\varkappa} .
$$

Proof. It is enough to consider the case of $q \neq 0$. Since the trigonometric polynomial $q$ is nonnegative, we see that the set $T=\left\{\alpha \in \mathbb{Z}^{\varkappa}: a_{\alpha} \neq 0\right\} \cup\{(0, \ldots, 0)\}$ satisfies the assumptions of Proposition 30 , and $q \in \mathscr{P}_{T}^{+}\left(\mathbb{T}^{\varkappa}\right)$. By this proposition, there exists $q_{\varepsilon} \in \Sigma_{T}^{2}\left(\mathbb{T}^{\varkappa}\right)$ such that $\left\|q-q_{\varepsilon}\right\|_{\text {co }}^{\prime} \leqslant \tilde{\varepsilon}$, where

$$
\tilde{\varepsilon}=\varepsilon \cdot \min \left\{1, \min \left\{\left|a_{\alpha}\right|: \alpha \in \mathbb{Z}^{\varkappa}, a_{\alpha} \neq 0\right\}\right\}>0 .
$$

This completes the proof.

For the case of $T=\mathbb{Z}^{\varkappa}$ it is well known that $\mathscr{P}_{\mathbb{Z}^{\varkappa}}^{+}\left(\mathbb{T}^{\varkappa}\right) \backslash \Sigma_{\mathbb{Z}^{\varkappa}}^{2}\left(\mathbb{T}^{\varkappa}\right) \neq \varnothing$ if $\varkappa \geqslant 2$ (cf. [46, page 51]). Recently Dritschel proved that 10 each (strictly) positive trigonometric polynomial in $\mathscr{P}\left(\mathbb{T}^{\varkappa}\right)$ belongs to $\Sigma_{\mathbb{Z}^{\varkappa}}^{2}\left(\mathbb{T}^{\varkappa}\right)$ (see [20, Corollary 5.2] where operator-valued trigonometric polynomials are considered; see also [27] for related questions concerning scalar trigonometric polynomials in two variables). The proof of Dritschel's result is based on his Theorem 5.1 which in the scalar case coincides with the major part of our Corollary 31 (except for (34)).

10 After posting the previous version of our paper in arXiv, preprint no. 0906.5410, K. Schmüdgen called our attention to the fact that the scalar version of Dritschel's result can be derived from the Archimedean Positivstellensatz (see e.g. 47. Corollary 3]). 
10. The truncated two-sided complex moment problem. Let $\mathfrak{Z}$ stand for the $*$-semigroup $(\mathbb{Z} \times \mathbb{Z},+, *)$ with coordinatewise defined addition as a semigroup operation and involution $(m, n)^{*}=(n, m)$. Given a subset $T$ of $\mathfrak{Z}$, we denote by $\Sigma_{T}^{2}\left(\mathbb{C}_{*}\right)$ the set of all rational functions $q \in \mathbb{C}_{T}(z, \bar{z})$ for which there exist finitely many rational functions $q_{1}, \ldots, q_{k} \in \mathbb{C}_{\mathfrak{Z}}(z, \bar{z})$ such that $q(z, \bar{z})=\sum_{j=1}^{k}\left|q_{j}(z, \bar{z})\right|^{2}$ for all $z \in \mathbb{C}_{*}$. Let $\mathscr{P}_{T}^{+}\left(\mathbb{C}_{*}\right)$ stand for the set of all rational functions $q \in \mathbb{C}_{T}(z, \bar{z})$ such that $q(z, \bar{z}) \geqslant 0$ for all $z \in \mathbb{C}_{*}$. We regard $\Sigma_{T}^{2}\left(\mathbb{C}_{*}\right), \mathscr{P}_{T}^{+}\left(\mathbb{C}_{*}\right)$ and $\mathbb{C}_{T}(z, \bar{z})$ as sets of complex functions on $\mathbb{C}_{*}$. Given $p \in \mathbb{C}_{\mathfrak{Z}}(z, \bar{z})$ of the form $p(z, \bar{z})=$ $\sum_{m, n \in \mathbb{Z}} a_{m, n} z^{m} \bar{z}^{n}$, we set $\|p\|_{\text {co }}=\max \left\{\left|a_{m, n}\right|: m, n \in \mathbb{Z}\right\}$. The function $\|\cdot\|_{\text {co }}$ is a norm on $\mathbb{C}_{\mathfrak{Z}}(z, \bar{z})$.

It was proved by Bisgaard (cf. [6]) that the $*$-semigroup $\mathfrak{Z}$ is semiperfect (in fact, as noted in [54, Theorem 24], $\mathfrak{Z}$ is operator semiperfect). This result enables us to apply our machinery. Below, we formulate counterparts of Theorem 19 (a shorter version), Proposition 28 and Corollary 29, Their proofs are analogical.

Theorem 32. Let $T$ be a symmetric subset of $\mathfrak{Z}$ (i.e. $(n, m) \in T$ for all $(m, n) \in T$ ) containing the diagonal ${ }^{11}\{(n, n): n \in \mathbb{Z}\}$, and let $Z \subset \mathbb{C}_{*}$ be a determining set for $\mathbb{C}[z, \bar{z}]$. Then for any system of complex numbers $\left\{c_{m, n}\right\}_{(m, n) \in T}$, the following conditions are equivalent:

(i) there exists a positive Borel measure $\mu$ on $\mathbb{C}_{*}$ such that

$$
c_{m, n}=\int_{\mathbb{C}_{*}} z^{m} \bar{z}^{n} \mu(\mathrm{d} z), \quad(m, n) \in T,
$$

(ii) $\sum_{(m, n) \in T} p_{m, n} c_{m, n} \geqslant 0$ for every finite system $\left\{p_{m, n}\right\}_{(m, n) \in T} \subset \mathbb{C}$ such that $\sum_{(m, n) \in T} p_{m, n} z^{m} \bar{z}^{n} \geqslant 0$ for all $z \in \mathbb{C}_{*}$,

(iii) $\sum_{(m, n) \in T} p_{m, n} c_{m, n} \geqslant 0$ for every finite system $\left\{p_{m, n}\right\}_{(m, n) \in T} \subset \mathbb{C}$ for which there exist finitely many rational functions $q_{1}, \ldots, q_{k} \in \mathbb{C}_{\mathfrak{Z}}(z, \bar{z})$ such that $\sum_{(m, n) \in T} p_{m, n} z^{m} \bar{z}^{n}=\sum_{j=1}^{k}\left|q_{j}(z, \bar{z})\right|^{2}$ for all $z \in Z$.

Proposition 33. Let $T$ be as in Theorem 32, Then the set $\Sigma_{T}^{2}\left(\mathbb{C}_{*}\right)$ is dense in $\mathscr{P}_{T}^{+}\left(\mathbb{C}_{*}\right)$ with respect to the finest locally convex topology on $\mathbb{C}_{T}(z, \bar{z})$. In particular, this is the case for the topology of uniform convergence on compact subsets of $\mathbb{C}_{*}$ and the topology given by the norm $\|\cdot\|_{\mathrm{co}}$.

Corollary 34. Let

$$
q(z, \bar{z})=\sum_{m, n \in \mathbb{Z}} a_{m, n} z^{m} \bar{z}^{n}, \quad z \in \mathbb{C}_{*},
$$

be a nonnegative rational function with coefficients in $\mathbb{C}$. Then for every $\varepsilon>0$, there exists a nonnegative rational function $q_{\varepsilon} \in \Sigma_{\mathfrak{Z}}^{2}\left(\mathbb{C}_{*}\right)$ such that

$$
\begin{aligned}
& q_{\varepsilon}(z, \bar{z})=\sum_{m, n \in \mathbb{Z}} a_{m, n}^{(\varepsilon)} z^{m} \bar{z}^{n}, \quad z \in \mathbb{C}_{*} \quad\left(a_{m, n}^{(\varepsilon)} \in \mathbb{C}\right), \\
& \left|a_{m, n}-a_{m, n}^{(\varepsilon)}\right| \leqslant \varepsilon\left|a_{m, n}\right|, \quad m, n \in \mathbb{Z}, m \neq n, \\
& \left|a_{m, n}-a_{m, n}^{(\varepsilon)}\right| \leqslant \varepsilon, \quad m, n \in \mathbb{Z} .
\end{aligned}
$$

Yet another proof of Proposition 33 consists in deriving it from Proposition 28. In turn, Corollary 34 can be deduced directly from Proposition 33. However, Theorem 32 does not seem to be an easy consequence of Theorem 19.

${ }^{11}$ Again, as noticed in footnote 9 this is the form that condition (13) now takes. 
11. Concluding remarks. A result of Bisgaard (cf. 6, 9]) states that the Cartesian product of two $*$-semigroups, one of which is (operator) perfect and the other (operator) semiperfect, is (operator) semiperfect. This fact can be applied to *semigroups $\mathbb{Z}^{\varkappa}$ (operator perfect) and $\mathfrak{N}_{+}$(operator semiperfect). Employing our method in this particular context we obtain appropriate results for mixed polynomials

$$
\sum_{\alpha \in \mathbb{Z}^{\varkappa}} \sum_{m, n \geqslant 0} a_{\alpha, m, n} w^{\alpha} z^{m} \bar{z}^{n}, \quad w \in \mathbb{T}^{\varkappa}, z \in \mathbb{C}
$$

Fortunately, there is a variety of $*$-semigroups which are semiperfect or operator semiperfect, and for which we can find a convenient description of their dual *semigroups and Laplace transforms (see e.g. [5, 12, 39, 50, 7, 8, 40, 22, 56]).

As we now show, a finitely generated $*$-semigroup with unit can be modelled as a quotient of the $*$-semigroup $\mathbb{Z}_{+}^{\varkappa} \times \mathbb{Z}_{+}^{\varkappa}$, which gives rise to yet another way of describing its dual. Suppose that a commutative $*$-semigroup $\mathfrak{S}$ with unit is finitely generated. Then there exists a finite system $\boldsymbol{e}=\left(e_{1}, \ldots, e_{\varkappa}\right)$ of elements of $\mathfrak{S}$ such that the mapping

$$
\Phi=\Phi_{\boldsymbol{e}}: \mathbb{Z}_{+}^{\varkappa} \times \mathbb{Z}_{+}^{\varkappa} \ni(\alpha, \beta) \mapsto \boldsymbol{e}^{\alpha} \boldsymbol{e}^{* \beta} \in \mathfrak{S}
$$

is a surjection, where $\boldsymbol{e}^{\alpha}=e_{1}^{\alpha_{1}} \ldots e_{\varkappa}^{\alpha_{\varkappa}}$ and $\boldsymbol{e}^{* \beta}=e_{1}^{* \beta_{1}} \ldots e_{\varkappa}^{* \beta_{\varkappa}}$ with $\alpha=\left(\alpha_{1}, \ldots, \alpha_{\varkappa}\right)$ and $\beta=\left(\beta_{1}, \ldots, \beta_{\varkappa}\right)$. Let us regard $\mathfrak{N}_{\varkappa} \stackrel{\text { def }}{=} \mathbb{Z}_{+}^{\varkappa} \times \mathbb{Z}_{+}^{\varkappa}$ as a $*$-semigroup with coordinatewise defined addition as a semigroup operation and involution $(\alpha, \beta)^{*}=(\beta, \alpha)$ for $\alpha, \beta \in \mathbb{Z}_{+}^{\varkappa}$. Then $\Phi$ is a $*$-epimorphism of $*$-semigroups, and consequently the relation $R=R_{\Phi}$ on $\mathfrak{N}_{\varkappa}$ defined by

$$
(\alpha, \beta) R\left(\alpha^{\prime}, \beta^{\prime}\right) \quad \text { if } \quad \Phi(\alpha, \beta)=\Phi\left(\alpha^{\prime}, \beta^{\prime}\right)
$$

is a congruence. As a consequence, the $*$-semigroup $\mathfrak{S}$ is $*$-isomorphic to the quotient $*$-semigroup $\mathfrak{N}_{\varkappa} / R$ (consult [32, Theorem I.1.5]). This means that the $*$ semigroups $\mathfrak{N}_{\varkappa} / R, \varkappa \geqslant 1$, are model $*$-semigroups in the category of finitely generated $*$-semigroups. We now describe the dual $*$-semigroup of the model $*$-semigroup $\mathfrak{N}_{\varkappa} / R$. In what follows, we regard $\mathbb{C}^{\varkappa}$ as a $*$-semigroup with coordinatewise defined multiplication as a semigroup operation and involution $\left(z_{1}, \ldots, z_{\varkappa}\right)^{*}=\left(\bar{z}_{1}, \ldots, \bar{z}_{\varkappa}\right)$. First, note that the set

$$
\mathfrak{F}_{R}=\left\{z \in \mathbb{C}^{\varkappa}: z^{\alpha} \bar{z}^{\beta}=z^{\alpha^{\prime}} \bar{z}^{\beta^{\prime}} \text { whenever }(\alpha, \beta) R\left(\alpha^{\prime}, \beta^{\prime}\right)\right\}
$$

is a $*$-subsemigroup of $\mathbb{C}^{\varkappa}$. It is a matter of routine to verify that the mapping

$$
\Psi: \mathfrak{F}_{R} \rightarrow \mathfrak{X}_{\mathfrak{N}_{\varkappa} / R}, \quad \Psi(z)\left([(\alpha, \beta)]_{R}\right)=z^{\alpha} \bar{z}^{\beta} \text { for }(\alpha, \beta) \in \mathfrak{N}_{\varkappa}, z \in \mathfrak{F}_{R},
$$

where $[(\alpha, \beta)]_{R}$ is the equivalence class of $(\alpha, \beta)$ with respect to the relation $R$, is a $*$-isomorphism of $*$-semigroups (hint: the mapping $\mathbb{C}^{\varkappa} \ni z \mapsto \varphi_{z} \in \mathfrak{X}_{\mathfrak{N}_{\varkappa}}$, where $\varphi_{z}(\alpha, \beta)=z^{\alpha} \bar{z}^{\beta}$ for $(\alpha, \beta) \in \mathfrak{N}_{\varkappa}$, is a $*$-isomorphism of $*$-semigroups). Applying the measure transport theorem to (35), one can rewrite the integrals $\int_{\mathfrak{X}_{\mathfrak{S}}} \hat{s} \mathrm{~d} \mu$ that appear in conditions (ii) of Theorems 14 and 15) in the form $\int_{\mathfrak{F}_{R}} z^{\alpha} \bar{z}^{\beta} \mathrm{d} \tilde{\mu}(z)$ which resembles the solution of the multidimensional complex moment problem. However, the example of the $*$-semigroup $\mathfrak{N}_{+}$in Section 5 shows that this general approach is not always efficient. 


\section{REFERENCES}

[1] J. R. Archer, Positivity and the existence of unitary dilations of commuting contractions, Oper. Theory Adv. Appl. 171 (2006), 17-35. MR2308555 (2008f:47011)

[2] E. Artin, Über die Zerlegung definiter Funktionen in Quadrate, Hamb. Abh. 5 (1927), 100-115; The collected papers of Emil Artin, 273-288, Addison-Wesley, 1965. MR0671416 (83j:01083)

[3] M. Bakonyi, The extension of positive definite operator-valued functions defined on a symmetric interval of an ordered group, Proc. Amer. Math. Soc. 130 (2002), 1401-1406. MR.1879963 (2003e:43005)

[4] M. Bakonyi, D. Timotin, Extensions of positive definite functions on free groups, J. Funct. Anal. 246 (2007), 31-49. MR2316876 (2008g:43009)

[5] C. Berg, J. P. R. Christensen, P. Ressel, Harmonic Analysis on Semigroups, Springer, Berlin, 1984. MR747302 (86b:43001)

[6] T. M. Bisgaard, The two-sided complex moment problem, Ark. Mat. 27 (1989), 23-28. MR 1004719 (91b:44015)

[7] T. M. Bisgaard, Extensions of Hamburger's theorem, Semigroup Forum 57 (1998), 397-429. MR.1640879 (99k:44015)

[8] T. M. Bisgaard, On perfect semigroups, Acta Math. Hungar. 79 (1998), 269-294. MR1619811 (99d:43006)

[9] T. M. Bisgaard, If $S \times T$ is semiperfect, is $S$ or T perfect? Hokkaido Math. J. 29 (2000), 523-529. MR1795489(2001i:43010)

[10] T. M. Bisgaard, Characterization of moment multisequences by a variation of positive definiteness, Collect. Math. 52 (2001), 205-218. MR.1885219(2002k:42017)

[11] T.M. Bisgaard, On the relation between the scalar moment problem and the matrix moment problem on *-semigroups, Semigroup Forum 68 (2004), 25-46. MR2027606 (2005b:43007)

[12] T. M. Bisgaard, P. Ressel, Unique disintegration of arbitrary positive definite functions on *-divisible semigroups, Math. Z. 200 (1989), 511-525. MR987584 (90b:43007)

[13] J. Bochnak, M. Coste, M.-F. Roy, Géometrie algébrique réelle, Ergeb. Math. Grenzgeb., vol. 12, Springer-Verlag, Berlin and New York, 1987. MR.949442 (90b:14030)

[14] R. Bruzual, M. Domínguez, Extension of locally defined indefinite functions on ordered groups, Integr. Equ. Oper. Theory 50 (2004), 57-81. MR2091054(2005g:47068)

[15] R. B. Burckel, Weakly Almost Periodic Functions on Semigroups, Gordon and Breach Science Publishers, New York-London-Paris, 1970. MR0263963 (41:8562)

[16] A.P. Calderón and R. Pepinsky, On the phase of Fourier coefficients for positive real periodic functions, in Computing Methods and the Phase Problem in X-ray Crystal Analysis, Department of Physics, Pennsylvania State College, State College, Pa. (1952), 339-348.

[17] D. Cichoń, J. Stochel, F. H. Szafraniec, Incomplete Riesz-Haviland criterion.

[18] A. H. Clifford, G. B. Preston, The algebraic theory of semigroups. Vol. I.Mathematical Surveys, No. 7, American Mathematical Society, Providence, R.I. 1961. MR0132791 (24:A2627)

[19] R. E. Curto, L. A. Fialkow, An analogue of the Riesz-Haviland theorem for the truncated moment problem, J. Funct. Anal. 255 (2008), 2709-2731. MR2464189 (2009i:47039)

[20] M. A. Dritschel, On factorization of trigonometric polynomials, Integr. Equ. Oper. Theory 49 (2004), 11-42. MR2057766 (2005d:47034)

[21] B. Fuglede, The multidimensional moment problem, Expo. Math. 1 (1983), 47-65. MR693807 (85g:44010)

[22] K. Furuta, N. Sakakibara, Radon perfectness of conelike *-semigroups in $Q^{(\infty)}$, Acta Math. Hungar. 91 (2001), 1-8. MR.1912357(2003d:22004)

[23] J.-P. Gabardo, Extension of positive-defnite distributions and maximum entropy, Memoirs of the Amer. Math. Soc. 489, Amer. Math. Soc., Providence, 1993. MR1139457 (93g:42005)

[24] J.-P. Gabardo, Tight frames of polynomials and the truncated trigonometric moment problem, J. Fourier Anal. Appl. 1 (1995), 249-279. MR1353540 (96m:42013)

[25] J.-P. Gabardo, Trigonometric moment problems for arbitrary finite subsets of $\boldsymbol{Z}^{n}$, Trans. Amer. Math. Soc. 350 (1998), 4473-4498. MR1443194 (99a:42005)

[26] J.-P. Gabardo, Truncated trigonometric moment problems and determinate measures, J. Math. Anal. Appl. 239 (1999), 349-370. MR1723065 (2000i:47028) 
[27] J. S. Geronimo, H. J. Woerdeman, Positive extensions, Fejér-Riesz factorization and autoregressive filters in two variables, Annals of Mathematics 160 (2004), 839-906. MR2144970 (2006b:42036)

[28] E. K. Haviland, On the momentum problem for distributions in more than one dimension, Amer. J. Math. 57 (1935), 562-568. MR1507095

[29] E. K. Haviland, On the momentum problem for distributions in more than one dimension, II, Amer. J. Math. 58 (1936), 164-168. MR.1507139

[30] E. Hewitt, K. A. Ross, Abstract Harmonic Analysis, Vol. II, Die Grundlehren der mathematischen Wissenschaften, Band 152, Springer-Verlag, New York-Berlin, 1970. MR0262773 $(41: 7378)$

[31] E. Hewitt, H. S. Zuckerman, The $l_{1}$-algebra of a commutative semigroup, Trans. Amer. Math. Soc. 83 (1956), 70-97. MR0081908 (18:465b)

[32] T. W. Hungerford, Algebra, Graduate Texts in Mathematics 73, Springer-Verlag, New York, 1974. MR600654 (82a:00006)

[33] Z. J. Jabłoński, J. Stochel, F. H. Szafraniec, Unitary propagation of operator data, Proceedings of the Edinburgh Mathematical Society 50 (2007), 689-699. MR2360524 (2008h:47022)

[34] Y. Kilpi, Über das komplexe Momentenproblem, Ann. Acad. Sci. Fenn. Ser.A. I. 236 (1957), 32 pp. MR0094660 (20:1172)

[35] M.G. Krein, Sur le problème du prolongement des fonctions hermitiennes positives et continues, Dokl. Akad. Nauk SSSR 26 (1940), 17-22 (Russian). MR.0004333(2:361h)

[36] R. J. Lindahl, P. H. Maserick, Positive-definite functions on involution semigroups, Duke Math. J. 38 (1971), 771-782. MR0291826 (45:916)

[37] P. H. Maserick, Spectral theory of operator-valued transformations, J. Math. Anal. Appl. 41 (1973), 497-507. MR0343084(49:7828)

[38] W. Mlak, Dilations of Hilbert space operators (General theory), Dissertationes Math. 153 (1978), 1-65. MR 496046(81j:47008)

[39] Y. Nakamura, N. Sakakibara, Perfectness of certain subsemigroups of a perfect semigroup, Math. Ann. 287 (1990), 213-220. MR1054564 (92b:43008)

[40] K. Nishio, N. Sakakibara, Perfectness of conelike *-semigroups in $\mathbf{Q}^{k}$, Math. Nachr. 216 (2000), 155-167. MR1774907 (2001g:43005)

[41] M. Putinar, F.-H. Vasilescu, Solving moment problems by dimensional extension, Ann. of Math. 149 (1999), 1087-1107. MR.1709313 (2001c:47023b)

[42] B. Reznick, Uniform denominators in Hilbert's seventeenth problem, Math. Z. 220 (1995), 75-97. MR:1347159 (96e:11056)

[43] M. Riesz, Sur le probléme des moments, Troisième Note, Arkiv für Matematik, Astronomi och Fysik 17 (1923), 1-52.

[44] W. Rudin, The extension problem for positive-definite functions, Illinois J. Math. 7 (1963), 532-539. MR0151796 (27:1779)

[45] W. Rudin, Functional analysis, McGraw-Hill Series in Higher Mathematics, McGraw-Hill Book Co., New York-Düsseldorf-Johannesburg, 1973. MR0365062 (51:1315)

[46] L. A. Sakhnovich, Interpolation Theory and its Applications, Kluwer Academic Publishers, Dordrecht, 1997. MR 1631843 (99j:47016)

[47] K. Schmüdgen, The $K$-moment problem for compact semi-algebraic sets, Math. Ann. 289 (1991), 203-206. MR1092173 (92b:44011)

[48] J. A. Shohat, J. D. Tamarkin, The problem of moments, Math. Surveys, vol II, Amer. Math. Soc., Providence, Rhode Island, 1943. MR0008438 (5:5c)

[49] J. Stochel, A note on general operator dilations over *-semigroups, Bull. Acad. Polon. Sci. Sér. Sci. Math. 28 (1980), 149-153. MR620351(83m:47008)

[50] J. Stochel, Moment functions on real algebraic sets, Ark. Mat. 30 (1992), 133-148. MR1171099 (93d:47032)

[51] J. Stochel and F. H. Szafraniec, On normal extensions of unbounded operators. I, J. Operator Theory 14 (1985), 31-55. MR789376 (87d:47034)

[52] J. Stochel and F. H. Szafraniec, On normal extensions of unbounded operators. II, Acta Sci. Math. (Szeged) 53 (1989), 153-177. MR1018684(91i:47032)

[53] J. Stochel, F. H. Szafraniec, On normal extensions of unbounded operators. III. Spectral properties, Publ. RIMS, Kyoto Univ. 25 (1989), 105-139. MR999353 (91i:47033)

[54] J. Stochel, F. H. Szafraniec, The complex moment problem and subnormality: A polar decomposition approach, J. Funct. Anal. 159 (1998), 432-491. MR.1658092 (2001c:47023a) 
[55] J. Stochel, F. H. Szafraniec, Unitary dilation of several contractions, Operator Theory: Adv. Appl. 127, Birkhäuser, Basel 2001, 585-598. MR1902903 (2003d:47008)

[56] J. Stochel, F. H. Szafraniec, Domination of unbounded operators and commutativity, J. Math. Soc. Japan 55 (2003), 405-437. MR.1961294 (2003m:47033)

[57] F. H. Szafraniec, A general dilation theorem, Bull. Acad. Polon. Sci., Sér. Sci. Math. Astr. et Phys. 25(1977), 263-267. MR0445309 (56:3651)

[58] F. H. Szafraniec, Boundedness of the shift operator related to positive definite forms: An application to moment problems, Ark. Mat. 19 (1981), 251-259. MR650498 (84b:44015)

[59] F. H. Szafraniec, Dilations of linear and nonlinear operator maps, Functions, series, operators (Budapest, 1980), 1165-1169, Colloq. Math. Soc. János Bolyai, 35, North-Holland, Amsterdam, 1983. MR.751074

[60] F. H. Szafraniec, Sur les fonctions admettant une extension de type positif, C. R. Acad. Sci. Paris 292 (1981), Sér. I, 431-432. MR611409 (82c:46075)

[61] B. Sz.-Nagy, Extensions of linear transformations in Hilbert space which extend beyond this space, Appendix to F. Riesz, B. Sz.-Nagy, Functional Analysis, Ungar, New York, 1960.

[62] B. Sz.-Nagy and C. Foiaş, Harmonic analysis of operators on Hilbert space, Akadémiai Kiadó, Budapest and North-Holland, Amsterdam, London, 1970. MR0275190 (43:947)

[63] F.-H. Vasilescu, Existence of unitary dilations as a moment problem, Oper. Theory Adv. Appl. 143 (2003), 333-344. MR2019357(2005h:47018)

Instytut Matematyki, Uniwersytet Jagielloński, Ul. ŁoJasiewicza 6, PL-30348 KraKÓw, POLAND

E-mail address: Dariusz.Cichon@im.uj.edu.pl

Instytut Matematyki, Uniwersytet Jagielloński, Ul. Łojasiewicza 6, PL-30348 KraKów, POLAND

E-mail address: Jan.Stochel@im.uj.edu.pl

Instytut Matematyki, Uniwersytet Jagielloński, Ul. ŁoJasiewicza 6, PL-30348 KraKÓw, POLAND

E-mail address: Franciszek.Szafraniec@im.uj.edu.pl 